An Improved Particle Filtering-based Approach for Health Prediction and Prognosis of Nonlinear Systems

Najmeh Daroogheh, Nader Meskin, Khashayar Khorasani

PII: S0016-0032(18)30145-5

DOI: 10.1016/j.jfranklin.2018.02.023

Reference: $\quad$ FI 3348

To appear in: $\quad$ Journal of the Franklin Institute

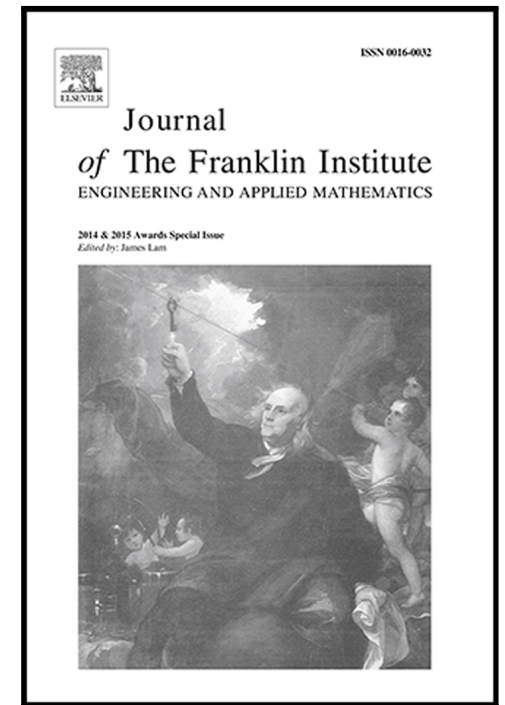

Received date: $\quad 20$ February 2017

Revised date: $\quad 10$ December 2017

Accepted date: $\quad 13$ February 2018

Please cite this article as: Najmeh Daroogheh, Nader Meskin, Khashayar Khorasani, An Improved Particle Filtering-based Approach for Health Prediction and Prognosis of Nonlinear Systems, Journal of the Franklin Institute (2018), doi: 10.1016/j.jfranklin.2018.02.023

This is a PDF file of an unedited manuscript that has been accepted for publication. As a service to our customers we are providing this early version of the manuscript. The manuscript will undergo copyediting, typesetting, and review of the resulting proof before it is published in its final form. Please note that during the production process errors may be discovered which could affect the content, and all legal disclaimers that apply to the journal pertain. 


\title{
An Improved Particle Filtering-based Approach for Health Prediction and Prognosis of Nonlinear Systems *
}

\author{
Najmeh Daroogheh ${ }^{1}$, Nader Meskin ${ }^{2}$ and Khashayar Khorasani ${ }^{1}$
}

\begin{abstract}
Health monitoring of nonlinear systems is broadly concerned with the system health tracking and its prediction to future time horizons. Estimation and prediction schemes constitute as principle components of any health monitoring technique. Particle filter (PF) represents a powerful tool for performing state and parameter estimation as well as prediction of nonlinear dynamical systems. Estimation of the system parameters along with the states can yield an up-to-date and reliable model that can be used for long-term prediction problems through utilization of particle filters. This feature enables one to deal with uncertainty issues in the resulting prediction step as the time horizon is extended. Towards this end, this paper presents an improved method to achieve uncertainty management for long-term prediction of nonlinear systems by using particle filters. In our proposed/approach, an observation forecasting scheme is developed to extend the system observation profiles (as time-series) to future time horizon. Particles are then propagated to future time instants according to a resampling algorithm instead of considering constant weights for the particles propagation in the prediction step. The uncertainty in the long-term prediction of the system states and parameters are managed by utilizing dynamic linear models for development of an observation forecasting scheme. This task is addressed through an outer adjustment loop for adaptively changing the sliding observation injection window based on the Mahalanobis distance criterion. Our proposed approach is then applied to predicting the health condition as well as the remaining useful life (RUL) of a gas turbine engine that is affected by degradations in the system health parameters. Extensive simulation and case studies are conducted to demonstrate and illustrate the capabilities and performance characteristics of our proposed and developed schemes.
\end{abstract}

\section{Index Terms}

Health monitoring, prognosis, particle filters, state and parameter prediction, observation forecasting, dynamically linear models, gas turbine engine, fouling, erosion.

\section{ACRONYMS AND ABBREVIATIONS}

DLM dynamic linear model

$\mathrm{PF} \quad$ particle filter

RUL remaining useful life

ARMA autoregressive moving average

PHM prognosis and health management

EF equivalent flop

PRMSE percent of root mean square error

RPE regularized prediction error

VOM variable order model

FOM fixed order model

W/O RS without resampling

\footnotetext{
${ }^{1}$ Najmeh Daroogheh and Khashayar Khorasani are with the Department of Electrical and Computer Engineering, Concordia University, Montreal, Quebec, Canada ndarooghe@gmail.com and kash@ece.concordia.ca

${ }^{2}$ Nader Meskin is with the Department of Electrical Engineering, Qatar University, Doha, Qatar nader.meskin@qu.edu.qa
} 
RUL-API RUL online accuracy-precision index

RUL-OPI RUL online precision index

RUL-OSI RUL online steadiness index

\section{Nomenclature:}
$x_{t} \quad$ system state
$\theta_{t} \quad$ fault vector of the system parameter
$\omega_{t} \quad$ process noise sequence
$\nu_{t} \quad$ measurement noise sequence
$\lambda\left(x_{t}\right) \quad$ system health parameter function
$\hat{y}_{t+i \mid t} \quad i$-step ahead observation prediction from the PF
$\hat{Y}_{t+i \mid t} \quad i$-step ahead observation forecast from the DLM
$y_{j, t}^{\operatorname{lin}} \quad$ the linear projection of the $j$-th observation
$\varepsilon_{j, t} \quad$ white noise process in the ARMA process for the $j$-th observation
$L \quad$ delay operator
$D_{\mathcal{M}} \quad$ Mahalanobis distance metric
$C($.$) \quad EF complexity$

\section{INTRODUCTION}

Reductions in maintenance cost is a challenging goal in today's highly complex and interconnected engineering systems. Highly relevant to this objective is prediction of the future health of a system. This problem has recently received a great deal of interest and is an active area of research for production and maintenance optimization goals. Research on reliable health monitoring techniques can potentially reduce the downtime and breakdowns of a system, and eonsequently add to the cost savings and operational safety [1], [2]. To achieve these goals, prognosis and health management (PHM) techniques have been pursued as principal active fields of research in various disciplines [3].

In PHM systems there are two principal components, namely: (i) the system health tracking that is to be achieved by analyzing the system behavior signatures or its health parameters (also known as the estimation module), and (ii) the system health prediction that is to be achieved by analyzing the evolution of system signatures in the long term horizon (also known as the prediction module) for predicting the system remaining useful life (RUL). Prognostic methods attempt to predict the future health of a system for determining its RUL before the failure occurs [1]. Performance of prognostic schemes is mainly influenced by accuracy of the prediction method, that in turn can be affected by activities such as the maintenance actions [4]. Therefore, uncertainty management is an important and challenging problem that should be considered in development of a reliable prognostic framework [5].

When the mathematical model of a system and its affected damage mechanisms are known, model-based prognostic methods can be utilized as proposed in the literature [6], [7]. Kalman filter based methods are used for performing prognosis in [8], [9], and a multiple model moving horizon estimation algorithm is developed for online prediction of the system health in [10]. Among all the model-based approaches for prognosis, particle filter (PF) Monte Carlo schemes are considered as representing the state-of-the-art in failure prognosis [5], [11], [12]. Their capability for taking into account and incorporating the system parameters as augmented states in the estimation scheme enables them to be suitable for uncertainty management in failure prognosis as achieved through joint state and parameter estimation modules. 
Development of a parameter estimation scheme for system health tracking when the damage model is not explicitly known was considered in [13], where the damage was identified according to its effect on the system health parameters. A dual state and parameter estimation method based on a nonlinear filtering scheme is developed in [13]. In this method, the time-varying health parameters of the system are estimated according to a novel approach based on the regularized prediction error (RPE) concept of particle filters (PF). In the present paper, the method developed in [14]-[16] is utilized to extend our previously developed dual state and parameter estimation algorithm for long-term prediction of system states and health parameters as required in our proposed prognosis scheme. Towards this end, an adaptive scheme for observation profile forecasting is proposed. This method is developed by incorporating the dynamic linear model (DLM) for Bayesian forecasting of univariate time-series [17], as opposed to nonlinear time-series forecasting methods that are developed in [18]. A statistical characterization of the profiles for Li-Ion batteries is addressed in [19] where a state space model is used to modify the obseryation equation that incorporates most of the static battery nonlinearities. This modification improves the convergence of the state estimate and provides suitable initial conditions for the prognosis stage.

The DLM represents a flexible approach for modeling a variety of fixed, time-varying, univariate or multivariate systems through Bayesian analysis [20]. The key feature in working with the DLM models is that their estimation and prediction schemes can be obtained recursiyely by using Bayesian approaches. One can generate useful models for forecasting non-stationary observations where the implementation and analysis are not as complicated as nonlinear time-series analytical methods that are used for forecasting [17], [20]. In the present work, DLM models and particle filters are integrated into an observation forecasting module for managing uncertainty in the long-term prediction of the system health condition. This is achieved by introducing fixed-lag DLM models that are updated according to an adaptive scheme. This adaptive updating scheme is developed based on the Mahalanobis distance metric that enables the prediction algorithm to manage uncertainties that are originated from the non-stationary process noise. The Mahalanobis distance is an important metric that has been used in the literature for fault diagnosis of dynamical systems [21].

An uncertainty management system for long-term prediction horizon using particle filters was developed in [5]. It utilizes invariant particle weights for future propagation of the particles. On the other hand, the regularization of particles is utilized to characterize the future uncertainties by modifying the location of particles. The prediction algorithm accuracy is shown to be improved by employing an outer correction loop to modify the hyper-parameters of the process noise that is used in the nonlinear model of the system. This is in particular implemented in the artificial evolution law that is utilized for estimating the system unknown parameters. An outer feedback correction loop in particle filtering based prognosis approaches has been introduced in several works [22]-[24]. Moreover, in a simpler prediction approach as far as computational effort is concerned in [5] the particle weights are considered fixed for future propagation. This is based on the assumption that the error generated by considering the invariant particle weights for future time instants are negligible when compared to other sources of error in practical applications affecting the system.

In our proposed method, it is shown that our previously developed dual state and parameter estimation algorithm in [14], [15] along with the newly developed DLM prediction method where the particles resampling is maintained for future time instants can yield an improved long-term prediction performance and achieve a more accurate RUL estimation of the system condition. These constitute as the main goals 
of the second component of any PHM strategy [22], [25]-[28]. Our results are evaluated by utilizing the percent of root mean square error criterion as well as the accuracy of the RUL prediction for the prognosis problem [29].

We have also conducted an extensive study on the computational complexity of our proposed method in terms of the so-called equivalent flop (EF) complexity that is introduced in [30] for any given operation. Moreover, the conditions under which the equivalent time complexity of our proposed method, including the observation prediction step and the resampling scheme for the prediction horizon, can be comparable to the previously developed methods in [5] with an augmented state and parameter vector and constant particle weight propagation, are also obtained. In this paper, our preliminary results in [13] are formally justified and validated through an extensive theoretical analysis as well as a comprehensive computational evaluation and quantitative comparisons.

Finally, our proposed prognosis strategy is applied to a gas turbine engine application to predict the system health parameters variations when it is subjected to soft degradation damages. The mathematical model of the considered gas turbine engine has been already verified and validated in our earlier works [31]-[33] by utilizing the commercially available software simulation toolbox GSP10 [34]. Based on the predicted system health parameters, the remaining useful life of the engine is determined. The main causes of degradation in a gas turbine engine can be categorized into (a) erosion, (b) corrosion, (c) fouling, and (d) thermal distortion that can initiate and/or accelerate the creep, low-cycle fatigue, high-cycle fatigue and thermal fatigue damage [35], [36]. In this work, we concentrate and mainly investigate the effects of fouling and erosion phenomena as the principle causes of the engine performance degradation. The probabilities of an engine failure due to these degradation phenomena are approximated based on our developed health prediction scheme.

To summarize, the main contributions of this paper can be stated as follows:

1) To develop an improved prediction strategy based on particle filters for performing the system failure prognosis,

2) To propose an observation forecasting scheme based on the fixed/variable order DLM that are adjusted online by utilizing an adaptive external adjustment mechanism,

3) To quantitatively determine and evaluate the computational complexity of our proposed methods in terms of the EF complexity, and

4) To apply our proposed and developed failure prognosis methodology to a highly nonlinear and complex case study of gas turbine engine subject to fouling and erosion degradations to comprehensively evaluate and demonstrate the advantages and capabilities of our proposed methodology.

The remainder of this paper is organized as follows. In Section II, the nonlinear filtering problem is formulated by including changes in the system health parameters through a multiplicative fault vector. A brief background information on our previously developed dual state and parameter nonlinear filtering schemes based on the particle filter method is also presented in this section. Our proposed framework for predicting the future propagation of the nonlinear system states and its parameters is developed in Section III. The utilization and implementation of our proposed prediction method in evaluating the system RUL is presented in Section IV. Section V provides the complexity analysis of our proposed prediction scheme based on the EF metric. Finally, extensive simulation results and comparative evaluation results using the EF and three RUL performance metrics corresponding to the application of our proposed method for failure prognosis of a gas turbine engine that is affected by degradations due to compressor fouling and 
turbine erosion are provided in Section VI.

\section{PROBLEM STATEMENT AND BACKGROUND INFORMATION}

In model-based prognosis and health monitoring approaches, the first step in formulating the problem is to characterize the damage model. Let us consider the following nonlinear dynamical system,

$$
\begin{gathered}
x_{t+1}=f_{t}\left(x_{t}, \theta_{t}^{\mathrm{T}} \lambda\left(x_{t}\right), \omega_{t}\right), \\
y_{t}=h_{t}\left(x_{t}, \theta_{t}^{\mathrm{T}} \lambda\left(x_{t}\right)\right)+\nu_{t},
\end{gathered}
$$

where $x_{t} \in \mathbb{R}^{n_{x}}$ is the system state, $\lambda():. \mathbb{R}^{n_{x}} \longrightarrow \mathbb{R}^{n_{\theta}}$ is a known differentiable function that determines the relationship between the health parameters and the system states, $\theta_{t} \in \mathbb{R}^{n_{\theta}}$ is an unknown and possibly time-varying multiplicative fault vector that represents the damage effects on the system health parameter where for the healthy system, $\theta_{t}$ is set to $\mathbf{1}, y_{t} \in \mathbb{R}^{n_{y}}$ is the output measurement, $\omega_{t} \in \mathbb{R}^{n_{\omega}}$ and $\nu_{t} \in \mathbb{R}^{n_{y}}$ are uncorrelated noise sequences with covariance matrices $W_{t}$ and $V_{t}$, and mean values $\mu_{w}$ and $\mu_{v}$, respectively (although the mean $\mu_{v} \equiv 0$ subsequently without loss of generality), $f_{t}: \mathbb{R}^{n_{x}} \times \mathbb{R} \times \mathbb{R}^{n_{\omega}} \longrightarrow \mathbb{R}^{n_{x}}$, and $h_{t}: \mathbb{R}^{n_{x}} \times \mathbb{R} \longrightarrow \mathbb{R}^{n_{y}}$ are known nonlinear functions representing the relationship between the states, parameters and the output measurements (observations). The degradation phenomenon in mechanical systems can in general be identified from the changes it causes on the efficiency of the system, where the efficiency is designated as the health parameter that can in general be analytically obtained from the states and measurements of the system. The process noise is not considered as an additive noise since corresponding to our main focused application problem (that is, a mechanical system aircraft gas turbine engine application) the additive process noise assumption is not necessarily valid.

\section{A. Background Information}

A brief, albeit required, background corresponding to the dual state and parameter estimation algorithm as has been developed in our earlier works in [13]-[16], is now presented in this section. In the developed dual state and parameter estimation structure, two concurrent filters are implemented for the state and parameter estimation tasks. At each time step for the state (parameter) estimator filter, the parameters (states) are treated as being known inputs to the filter from the parameter (state) estimator.

Our main goal in the dual state and parameter estimation problem is to approximate the following conditional expectations

$$
\begin{aligned}
\mathbb{E}\left(\phi_{1}\left(x_{t}\right) \mid y_{1: t}, \theta_{t-1}\right) & =\int \phi_{1}\left(x_{t}\right) p\left(x_{t} \mid y_{1: t}, \theta_{t-1}\right) \mathrm{d} x_{t}, \\
\mathbb{E}\left(\phi_{2}\left(\theta_{t}\right) \mid y_{1: t}, x_{t}\right) & =\int \phi_{2}\left(\theta_{t}\right) p\left(\theta_{t} \mid y_{1: t}, x_{t}\right) \mathrm{d} \theta_{t},
\end{aligned}
$$

where $y_{1: t}=\left(y_{1}, y_{2}, \ldots, y_{t}\right)$ denotes the available observations up to the time instant $t, \phi_{1}: \mathbb{R}^{n_{x}} \rightarrow \mathbb{R}$, and $\phi_{2}: \mathbb{R}^{n_{\theta}} \rightarrow \mathbb{R}$ are functions of states and parameters, respectively, that are to be estimated. The conditional probability functions $p\left(x_{t} \mid y_{1: t}, \theta_{t-1}\right) \mathrm{d} x_{t}$ and $p\left(\theta_{t} \mid y_{1: t}, x_{t}\right) \mathrm{d} \theta_{t}$ are to be approximated by the designed particle filters (PFs) through determining the filtering distributions according to,

$$
\begin{aligned}
\hat{p}_{N}\left(x_{t} \mid y_{1: t}, \theta_{t-1}\right) \mathrm{d} x_{t} & =\sum_{i=1}^{N} w_{x_{t}}^{(i)} \delta_{x_{t}^{(i)}}\left(\mathrm{d} x_{t}\right), \\
\hat{p}_{N}\left(\theta_{t} \mid y_{1: t}, x_{t}\right) \mathrm{d} \theta_{t} & =\sum_{j=1}^{N} w_{\theta_{t}}^{(j)} \delta_{\theta_{t}^{(j)}}\left(\mathrm{d} \theta_{t}\right),
\end{aligned}
$$


- Initialization of the states and parameters: $x_{0} \sim \pi_{x_{0}}$ and $\theta_{0} \sim \pi_{\theta_{0}}$, where $\pi_{x_{0}}$ and $\pi_{\theta_{0}}$ are the initial distributions of states and parameters, respectively,

$\left[\hat{x}_{t \mid t},\left\{\hat{x}_{t \mid t}^{(i)}\right\}_{i=1}^{N}, \hat{\theta}_{t \mid t},\left\{\hat{\theta}_{t \mid t}^{(j)}\right\}_{j=1}^{N}, \Sigma_{\hat{\theta}_{t \mid t}}\right]=\operatorname{DualPF}\left(\left\{\hat{x}_{t-1 \mid t-1}^{(i)}\right\}_{i=1}^{N},\left\{\hat{\theta}_{t-1 \mid t-1}^{(j)}\right\}_{j=1}^{N}, \Sigma_{\hat{\theta}_{t-1 \mid t-1}}, y_{t}\right)$

1) Estimation of the a priori state distribution:

a) Generate the a priori state particles: $\hat{x}_{t \mid t-1}^{(i)}=f_{t}\left(\hat{x}_{t-1 \mid t-1}^{(i)}, \hat{\theta}_{t-1 \mid t-1}^{\mathrm{T}} \lambda\left(\hat{x}_{t-1 \mid t-1}^{(i)}\right), \omega_{t}^{(i)}\right), i=1, \ldots, N$, where $\omega_{t}^{(i)}$ follows the same distribution as $\omega_{t}$ in (1),

b) A priori state covariance approximation: $\Sigma_{\hat{x}_{t \mid t-1}}=\frac{1}{N-1} \sum_{i=1}^{N}\left(\hat{x}_{t \mid t-1}^{(i)}-\frac{1}{N} \sum_{j=1}^{N} \hat{x}_{t \mid t-1}^{(j)}\right)\left(\hat{x}_{t \mid t-1}^{(i)}-\frac{1}{N} \sum_{j=1}^{N} \hat{x}_{t \mid t-1}^{(j)}\right)^{\mathrm{T}}$.

2) Estimation of the a posteriori state distribution:

a) Calculate the state particles weights: $\tilde{w}_{x_{t}}^{(i)} \triangleq \frac{\rho\left(y_{t} \mid \hat{x}_{t \mid t-1}^{(i)}, \hat{\theta}_{t-1 \mid t-1}\right)}{\sum_{i=1}^{N} \rho\left(y_{t} \mid \hat{x}_{t \mid t-1}^{(i)}, \hat{\theta}_{t-1 \mid t-1}\right)}, i=1, \ldots, N$ where $\rho\left(y_{t} \mid.\right)$ is the conditional probability density function of $y_{t}$,

b) Regularization of the a priori state distribution: $\tilde{\pi}_{x_{t \mid t}}^{N}\left(\mathrm{~d} x_{t}\right) \approx \sum_{l=1}^{N_{\text {reg }}} \sum_{i=1}^{N} \tilde{w}_{x t}^{(i)} \frac{\left|\mathbb{A}_{t}^{-1}\right|}{b^{n} x} \mathcal{K}\left(\frac{1}{b} \mathbb{A}_{t}^{-1}\left(x_{t}^{\text {regl }}-\hat{x}_{t \mid t-1}^{(i)}\right)\right)$, where $\mathbb{A}_{t}$ is selected such that $\mathbb{A}_{t} \mathbb{A}_{t}^{\mathrm{T}}=\Sigma_{\hat{x}_{t \mid t-1}}$

c) Resampling to approximate a posteriori state estimate distribution: $\pi_{x_{t \mid t}}^{N}\left(\mathrm{~d} x_{t}\right)=\frac{1}{N} \sum_{i=1}^{N} \delta_{\hat{x}_{t \mid t}^{(i)}}\left(\mathrm{d} x_{t}\right)$,

d) Estimate the a posteriori state: $\hat{x}_{t \mid t}=\frac{1}{N} \sum_{i=1}^{N} \hat{x}_{t \mid t}^{(i)}$.

3) Estimation of the parameter distribution:

a) Calculate the prediction error as: $\epsilon_{t}\left(\hat{\theta}_{t-1 \mid t-1}^{(j)}\right)=y_{t}-h\left(\hat{x}_{t \mid t}, \hat{\theta}_{t-1 \mid t-1}^{(j)^{\mathrm{T}}} \lambda\left(\hat{x}_{t \mid t}\right)\right), j=1, \ldots, N$,

b) Calculate $m_{t}^{(j)}$ from the RPE-based modified artificial law: $m_{t}^{(j)}=\hat{\theta}_{t-1 \mid t-1}^{(j)}+\gamma_{t} R_{t}^{(j)} \psi_{t}^{(j)} \epsilon_{t}\left(\hat{\theta}_{t-1 \mid t-1}^{(j)}\right), j=1, \ldots, N$,

c) Apply the kernel smoothing concept through the shrinkage matrix $A$ to obtain the first step a posteriori parameter estimation distribution: $\tilde{\theta}_{t \mid t}^{(j)}=A m_{t}^{(j)}+(I-A) \bar{m}_{t-1}+\zeta_{t}^{(j)}, \bar{m}_{t-1}=\frac{1}{N} \sum_{j=1}^{N} \hat{\theta}_{t-1 \mid t-1}^{(j)}, \zeta_{t}^{(j)} \sim \mathcal{N}\left(0,\left(I-A^{2}\right) \Sigma_{\hat{\theta}_{t-1 \mid t-1}}\right)$,

4) Estimation of the a posteriori parameter distribution:

a) Calculate the parameter particles weights: $\tilde{w}_{\theta_{t}}^{(j)} \triangleq \frac{\rho\left(y_{t}\left|\hat{x}_{t \mid t}\right| \tilde{\theta}_{t|t|}^{(j)}\right)}{\sum_{j=1}^{N} \rho\left(y_{t} \mid \hat{x}_{t \mid t} \tilde{\theta}_{t \mid t}^{(j)}\right)}, j=1, \ldots, N$,

b) Calculate the first step a posteriori parameter estimation distribution: $\tilde{\pi}_{\theta_{t \mid t}}^{N}\left(\mathrm{~d} \theta_{t}\right) \approx \sum_{j=1}^{N} \tilde{w}_{\theta_{t}}^{(j)} \delta_{\tilde{\theta}_{t \mid t}^{(j)}}\left(\mathrm{d} \theta_{t}\right)$,

c) Resampling to approximate a posteriori parameter estimate distribution: $\pi_{\theta_{t \mid t}}^{N}\left(\mathrm{~d} \theta_{t}\right)=\frac{1}{N} \sum_{j=1}^{N} \delta_{\hat{\theta}_{t \mid t}^{(j)}}^{t \mid t}\left(\mathrm{~d} \theta_{t}\right)$,

d) Obtain the a posteriori parameter estimate: $\hat{\theta}_{t \mid t}=\frac{1}{N} \sum_{j=1}^{N} \hat{\theta}_{t \mid t}^{(j)}$,

e) Calculate the a posteriori parameter estimate covariance: $\Sigma_{\hat{\theta}_{t \mid t}}=\frac{1}{N-1} \sum_{j=1}^{N}\left(\hat{\theta}_{t \mid t}^{(j)}-\hat{\theta}_{t \mid t}\right)\left(\hat{\theta}_{t \mid t}^{(j)}-\hat{\theta}_{t \mid t}\right)^{\mathrm{T}}$.

The definition of the notations in the pseudo code is as follows:

- $\omega_{t}^{(i)}$ denotes the process noise added to each particle for $i=1, \ldots, N$,

- $\mathcal{K}($.$) : The regularization kernel,$

- $x_{t}^{\text {reg }}$ : The regularized points around which $\mathcal{K}($.$) is evaluated for l=1, \ldots, N_{\text {reg }}$ (number of regularized steps),

- $\gamma_{t} R_{t}^{(j)}$ : The adaptive step size in the parameter estimation where $R_{t}^{(j)}=\sqrt{\operatorname{trace}\left(\mathcal{E}_{t}^{(j)} \mathcal{E}_{t}^{(j)^{\mathrm{T}}}\right)}, \mathcal{E}_{t}^{(j)}=\epsilon_{t}\left(\hat{\theta}_{t-1 \mid t-1}^{(j)}\right)-$ $\frac{1}{n_{y}} \sum_{l=1}^{l=n_{y}} \epsilon_{t}^{(l)}\left(\hat{\theta}_{t-1 \mid t-1}^{(j)}\right)$, where $\epsilon_{t}^{(l)}\left(\hat{\theta}_{t-1 \mid t-1}^{(j)}\right)$ denotes the $l$-th element of the $\epsilon_{t}\left(\hat{\theta}_{t-1 \mid t-1}^{(j)}\right)$ vector, and $\gamma_{t}$ is a constant or a decreasing step size,

- $\psi_{t}^{(j)}$ : The Jacobian of $h\left(\hat{x}_{t \mid t}, \hat{\theta}_{t-1 \mid t-1}^{(j)}\right)$ with respect to $\hat{\theta}_{t-1 \mid t-1}^{(j)}$ evaluated for the $j$-th particle,

- $\zeta_{t}^{(j)}$ : The evolution noise added to each parameter particle.

- $A$ : The shrinkage matrix is selected such that $A \leq I$.

where the subscript $N$ implies that the state and parameter conditional probability distributions are obtained from $N$ particles. Each state particle $x_{t}^{(i)}$ is associated with a weight $w_{x_{t}}^{(i)}$ and each parameter particle $\theta_{t}^{(j)}$ is associated with a related weight $w_{\theta_{t}}^{(j)}$, where $\delta($.$) denotes the Dirac-delta function mass. In Table I, the$ pseudo code corresponding to our previously developed dual state and parameter estimation filter for the nonlinear system (1)-(2) is provided. More details regarding this algorithm can be found in [14]-[16].

We are now in a position to present our proposed particle filters-based prediction framework by utilizing DLMs acting as local models to obtain and develop observation profiles forecasting. 


\section{PRediction Framework}

In this section, our proposed model-based prognosis approach (that is, the system health prediction), as described in Section II, is developed and presented. This is accomplished by extending our previously developed PF-based dual state and parameter estimation scheme [13]-[16] to the future time instants, where the weight update in the long-term prediction using particle filters cannot be easily implemented in absence of future observations.

Our proposed strategy is based on first forecasting the system observations by using the available historical data for a given predefined time horizon. This window is adaptively adjusted whenever a new observation batch becomes available. However, due to the system nonlinearity, the non-normality of measurement noise, and degradation effects, these will generally lead to non-stationary system observations [37]. Consequently, to model dynamical system observations as a time-series, as opposed to directly using a nonlinear model, the dynamic linear model (DLM) approach is utilized. The DLM is constructed based on the assumptions of local normality and linearity of the time-series data within a given short duration time interval in which the observation time-series has a stationary behavior manifestation [37].

The DLM model essentially represents a special class of state, space linear and Gaussian models, in which the time-series is considered as the output of a dynamical system that is stimulated by random disturbances. In our proposed fixed-lag DLM model, in each time window the available observation history that originates from a stochastic non-stationary process is approximated by a stationary process based on a linear regression method, and the observation forecasting is performed corresponding to a specific time horizon window.

By augmenting and integrating the observation forecasting module with the PF-based estimation scheme, the PF algorithm can be extended to future time horizons by utilizing the same weight update rule (through performing a resampling procedure). This is accomplished in the same manner as in the estimation module for predicting the system state and parameters for a long-term horizon according to the previously stated estimation algorithm (Section II-A)

The DLM structure for observation forecasting is constructed according to the well-known autoregressive moving average (ARMA) models. In the next subsection, a brief overview to forecasting with ARMA models within the DLM formulation is provided.

\section{A. Forecasting with ARMA Models}

ARMA models are well-known as suitable modeling strategies for forecasting or predicting stationary zero-mean stochastic processes. Although, the observation process $y_{t}$ in (2) is non-stationary, one can still approximate it by an ARMA process using the DLM in a short-term time window. It is assumed that variations in the observation time-series are not very fast during this time window, so that the assumption of the stationarity remains valid. This assumption is indeed not going to be restrictive at all as far as degradations forecasting in dynamical systems are concerned, since the degradation affects the system dynamical behavior quite slowly in time. Next, we analyze the specifications for the ARMA models that are to be utilized in our developed fixed-lag DLM framework.

Towards the above end, let us denote $y_{j, t}, j=1, \ldots, n_{y}$ as the $j$-th element of the output measurement vector $y_{t}$ (implying a univariate time-series). Since $y_{t}$, and consequently $y_{j, t}$, are non-stationary processes, to model each $y_{j, t}$ as a stationary time-series one has to consider a time interval during which the process behavior can be approximated as a stationary process. It is assumed that $y_{j, t}$ is a stationary process 
between the time intervals $t_{l}$ and $t_{l+1}$, where $l \in \mathbb{N}$ and $\tau$ denotes the number of available data points within the time window $\left(t_{l}-\tau, t_{l}\right]$. These data points are used for constructing the $l$-th DLM model corresponding to each output in the interval $\left[t_{l}, t_{l+1}\right)$. Subsequently, we will show how to select and specify the time instants $t_{l}, l \in \mathbb{N}$ based on the Mahalanobis distance criterion [38].

The DLM models corresponding to our observation forecasting module are constructed according to the ARMA model. Consequently, corresponding to each specific output $j$ in the time interval $\left(t_{l}-\tau, t_{l}\right]$, the $\operatorname{ARMA}\left(p_{j}, q_{j}\right)$ structure is selected and specified as follows,

$$
\begin{array}{r}
\left(1-\phi_{j, l, 1} L-\phi_{j, l, 2} L^{2}-\ldots-\phi_{j, l, p_{j}} L^{p_{j}}\right)\left(y_{j, t}-\mu_{j, l}\right)= \\
\left(1+\theta_{j, l, 1} L+\theta_{j, l, 2} L^{2}+\ldots+\theta_{j, l, q_{j}} L^{q_{j}}\right) \varepsilon_{j, t},
\end{array}
$$

where $\phi_{j, l, i}, i=1, \ldots, p_{j}$, and $\theta_{j, l, i}, i=1, \ldots, q_{j}$ denote the autoregressive and the moving average coefficients, respectively, the $p_{j}$ and $q_{j}$ denote the autoregressive and the moving average delay orders, respectively, $L$ denotes the delay operator, $\mu_{j, l}$ denotes the mean of the $j$-th historical observation $y_{j, t}$ in the time window $\left(t_{l}-\tau, t_{l}\right]$, and the sequence $\left\{\varepsilon_{j, t}\right\}$ is a white noise error process having a zero mean and a variance $\sigma_{\varepsilon_{j}}$.

Remark 1: The order of $\operatorname{ARMA}\left(p_{j}, q_{j}\right)$ can be different corresponding to any given $l$-th DLM model related to each observation time-series $y_{j, t}$. Therefore, the DLM model can be implemented based on a fixed order or a variable order ARMA process. In the case of a variable order ARMA model, the wellknown Akaike information criterion [39] can be utilized to determine the order of the ARMA process.

Another representation for the ARMA process in (3) can be obtained as follows,

$$
y_{j, t}-\mu_{j, l}=\frac{\left(1+\theta_{j, l, 1} L+\theta_{j, l, 2} L^{2}+\ldots+\theta_{j, l, q_{j}} L^{q_{j}}\right)}{\left(1-\phi_{j, l, 1} L-\phi_{j, l, 2} L^{2}, \ldots \ldots-\phi_{j, l, p_{j}} L^{p_{j}}\right)} \varepsilon_{j, t},
$$

which yields,

$$
y_{j, t}-\mu_{j, l}=\psi_{j, l, 1} \varepsilon_{j, t}+\psi_{j, l, 2} \varepsilon_{j, t-1}+\cdots,
$$

where $\psi_{j, l, i}, i=1,2, \ldots$ denotes the coefficients corresponding to the quotient of the term in the right hand side of (4), and refer to the fact that the stationary process $y_{j, t}-\mu_{j, l}$ can be written as an infinite autoregressive problem [37]. Therefore, the realization of (5) at the instant $t+1$ based on the information set $\left\{\varepsilon_{j, t+1}, \varepsilon_{j, t}, \varepsilon_{j, t-1}, \cdots\right\}$ is obtained as $y_{j, t+1}-\mu_{j, l}=\psi_{j, l, 1} \varepsilon_{j, t+1}+\psi_{j, l, 2} \varepsilon_{j, t}+\cdots$. Now, let us define a forecasting function that is based on the information set up to the time $t$, i.e. $\left\{\varepsilon_{j, t}, \varepsilon_{j, t-1}, \cdots\right\}$ as $\hat{Y}_{j, t+1 \mid t}-\mu_{j, l}=\rho_{j, l, 1} \varepsilon_{j, t}+\rho_{j, l, 2} \varepsilon_{j, t-1}+\cdots$. It has been clearly shown in the literature [18], [37], [40] that the mean-square error which minimizes $\mathbb{E}\left\{\left(y_{j, t+1}-\hat{Y}_{j, t+1 \mid t}\right)^{2}\right\}$ is achieved by setting $\rho_{j, l, i}=\psi_{j, l, i}$. This can also be derived from (3) which generates the true value of $y_{j, t+1}$ by setting the unobserved noise $\varepsilon_{j, t+1}$ to zero. Therefore, the one-step ahead forecast at time $t$ using the ARMA process (3) model is now obtained as,

$$
\begin{aligned}
\hat{Y}_{j, t+1 \mid t}-\mu_{j, l} & =\phi_{j, l, 1}\left(y_{j, t}-\mu_{j, l}\right)+\phi_{j, l, 2}\left(y_{j, t-1}-\mu_{j, l}\right)+ \\
& \ldots+\phi_{j, l, p_{j}}\left(y_{j, t-p_{j}+1}-\mu_{j, l}\right) \\
& +\theta_{j, l, 1} \varepsilon_{j, t}+\theta_{j, l, 2} \varepsilon_{j, t-1}+\ldots+\theta_{j, l, q_{j}+1} \varepsilon_{j, t-q_{j}+1} .
\end{aligned}
$$

The ARMA model parameters can be estimated by applying any recursive parameter estimation method, such as the least-mean squares (LMS), the recursive-least squares (RLS) or the Kalman filters [41] such that the desired cost function $V_{\tau}\left(\phi_{j, l, n}, \theta_{j, l, m}\right)=\frac{1}{\tau} \sum_{t=t_{l}-\tau+1}^{t_{l}}\left(y_{j, t}-\hat{Y}_{j, t \mid t-1}\right)^{2}$ is minimized given the available data within the time window $t \in\left(t_{l}-\tau, t_{l}\right]$, where $n=1, \ldots, p_{j}$ and $m=1, \ldots, q_{j}$. 
We first utilize this stationary process to construct the locally dynamic linear model that is represented by the ARMA process. Subsequently, the observation forecast will be performed given the obtained DLM model. The window size $\tau$ refers to a fixed number of available data points that are used to construct the DLM model. As new observations become available, the DLM model will be updated according to the Mahalanobis distance [38] through an external adjustment loop. In general, the observation forecasting task is accomplished recursively for the window time interval $[t+1, t+k]$, and is denoted by $\hat{Y}_{t+i \mid t}=\left(\hat{Y}_{1, t+i \mid t}, \ldots, \hat{Y}_{n_{y}, t+i \mid t}\right)^{\mathrm{T}}, i=1, \ldots, k$. More details regarding the DLM update procedure are investigated below.

1) The DLM Update Law: As stated earlier, at the instant $t=t_{l}$ a new DLM model is constructed based on the available data within the interval $\left(t_{l}-\tau, t_{l}\right]$. The next step deals with determining when the selected DLM should be updated. It is assumed that a new observation data batch are received with the size $s$, where the minimum value of $s$ is 1 and its maximum value depends on the size of the observation data batch that becomes available at each step. For example, for an aircraft engine the number of recorded data at each flight cycle might be more than one data point for each measurement. Therefore, at $t=t_{l}+s$, it is required to check the validity of the stationarity assumption.

The validation data set corresponds to the available observations in the time interval $\left[t_{l}+s-s+1, t_{l}+s\right]$, where $s$ is the size of the validation data. To check changes in the observation data, a sliding window of size $q$ is moved over the validation data and the Mahalanobis distance metric [38] is used to detect the change as follows. With reference to Figure 1, let us define two q-tuples which determine the two sliding windows in the calculation of the Mahalanobis distance metric corresponding to the observation vector as $Q_{i, 1}=\left(y_{t_{l}+s-\dot{s}+i}, \ldots, y_{t_{l}+s-\dot{s}+\dot{q}+i-1}\right)^{T}$ and $Q_{i, 2}=\left(y_{t_{l}+s-s+i+1}, \ldots, y_{t_{l}+s-\dot{s}+\dot{q}+i}\right)^{T}$ for $i=1, \ldots, \dot{s}-\dot{q}$. Next, the mean of data for these two vectors are calculated as,

$$
\mu_{Q_{i, 1}}=\frac{1}{\dot{q}} \sum_{j=0}^{\dot{q}-1} y_{t_{l}-s-\dot{s}+i+j}, \quad \mu_{Q_{i, 2}}=\frac{1}{\dot{q}} \sum_{j=0}^{\dot{q}-1} y_{t_{l}+s-\dot{s}+i+1+j},
$$

and the corresponding covariance matrices are denoted by $\Sigma_{Q_{i, 1}}$ and $\Sigma_{Q_{i, 2}}$, so that the Mahalanobis distance is calculated for the data points in $Q_{i, 1}$ and $Q_{i, 2}$ as follows,

$$
\begin{aligned}
& D_{\mathcal{M}}\left(Q_{i, 1}\right)=\sqrt{\left(Q_{i, 1}-\mu_{Q_{i, 1}} \mathbf{1}\right)^{\mathrm{T}} \Sigma_{Q_{i, 1}}^{-1}\left(Q_{i, 1}-\mu_{Q_{i, 1}} \mathbf{1}\right)} \\
& D_{\mathcal{M}}\left(Q_{i, 2}\right)=\sqrt{\left(Q_{i, 2}-\mu_{Q_{i, 2}} \mathbf{1}\right)^{\mathrm{T}} \Sigma_{Q_{i, 2}}^{-1}\left(Q_{i, 2}-\mu_{Q_{i, 2}} \mathbf{1}\right)}
\end{aligned}
$$

where $D_{\mathcal{M}}($.$) denotes the Mahalanobis distance for the observation vector and \mathbf{1}$ denotes a unit column vector. The change in the observation data at the time instant $t_{l}+i$ is detected if the following condition is satisfied according to [38], [42], as $\exists i \in\{2, \ldots, \dot{s}-\dot{q}\}$ such that $\left|D_{\mathcal{M}}\left(Q_{i, 1}\right)-D_{\mathcal{M}}\left(Q_{i, 2}\right)\right|>\delta \mid D_{\mathcal{M}}\left(Q_{i-1,1}\right)-$ $D_{\mathcal{M}}\left(Q_{i-1,2}\right) \mid$, where $\delta \geq 1$ is a positive constant. This along with $\left|D_{\mathcal{M}}\left(Q_{i-1,1}\right)-D_{\mathcal{M}}\left(Q_{i-1,2}\right)\right|$ determine the threshold for the change detection and its determination is application specific and the window length $\dot{s}$, and $\dot{q}$ are chosen such that $s-q \dot{q}>2$. Once the change is detected in observations at the time instant $t_{l}+i$, then $t_{l+1}=t_{l}+i$ and the new DLM model is obtained based on observations in the time interval $\left(t_{l+1}-\tau, t_{l+1}\right]$, otherwise the DLM model is not updated. However, a validation algorithm is performed whenever a new $s$ observation data become available, therefore it is executed at the time instant $t_{l}+\dot{k} s$, where $k$ denotes the number of data batches received after the last DLM update. The schematic depiction of our proposed DLM update algorithm based on the Mahalanobis distance is provided in Figure 1 on a typical trajectory of $y_{j, t}$. 


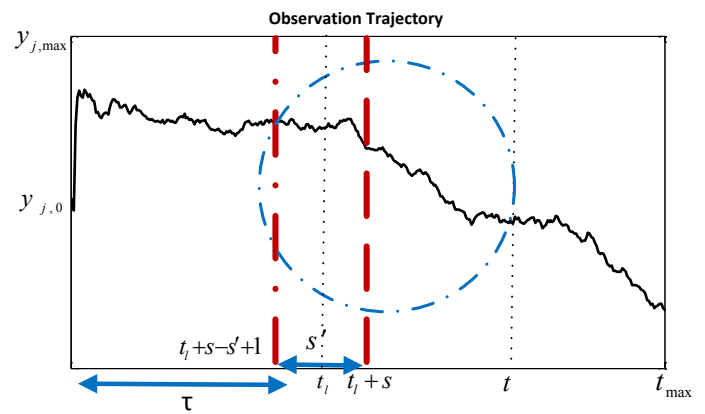

(a) Observation trajectory.
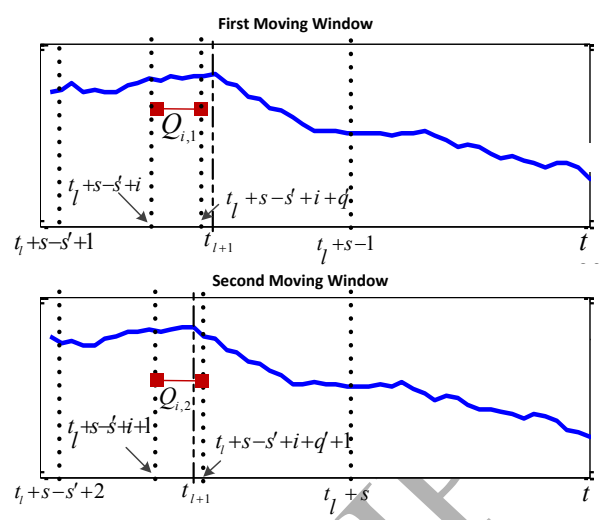

(b) Magnified change in the observation trajectory under two moving windows.

Fig. 1. Change detection in a sample observation trajectory for the DLM update.

Remark 2: The Mahalanobis distance metric for determining when the DLM model should be updated can also be defined based on the error between the predicted observations from the DLM model and the real observations that are available in the time window $\left(t_{l}, t_{l}+s\right]$ as in [13]. In such a case, more data will be required for evaluating the error based on this criterion.

Remark 3: It should be noted that the Mahalanobis distance metric takes into account not only the changes in the mean value of $y_{i, t}$ data, but also changes in the covariance of the observation data in the predefined time frame. Therefore, as soon as changes in these two parameters are detected the new observation data set is calculated and utilized to construct the DLM model. However, in the degradation phenomenon (unlike the case of an abrupt fault occurrence) changes in the system health parameters, and as a result in the system output observations, are quite gradual and slow and the stationary assumption within a limited and short time interval and window is indeed a valid assumption. The Mahalanobis distance (M-Distance) metric actually compensates for the non-stationarity of observations by moving the observation window towards the data set which is matched more to the stationary $y_{i, t}$ data set.

\section{B. Fixed-Lag DLM Model Error Analysis for Observation Forecasting}

An error analysis corresponding to the observation forecasting scheme is now presented in this subsection. Based on the given ARMA $\left(p_{j}, q_{j}\right)$ model in (3), the $l$-th general univariate DLM (for the $j$-th output of the dynamical system) can be written according to the following state space representation [17],

$$
\begin{aligned}
& \mathcal{Y}_{j, t}=G_{j, l} \mathcal{Y}_{j, t-1}+F_{j, l} \varepsilon_{j, t}, \\
& y_{j, t}-\mu_{j, l}=H \mathcal{Y}_{j, t},
\end{aligned}
$$

where $y_{j, t}$ for $t \in\left(t_{l}-\tau, t_{l}\right]$ refers to the available observations in the time interval of size $\tau, d_{j}=$ $\max \left(p_{j}, q_{j}+1\right), F_{j, l}=\left(\begin{array}{l}1 \\ \theta_{j, l, 1} \ldots \theta_{j, l, d_{j}-1}\end{array}\right)^{\mathrm{T}}, H=\left(\begin{array}{lll}1 & 0 \ldots 0\end{array}\right), \mathcal{Y}_{j, t}=\left(y_{j, t}-\mu_{j, l}, y_{j, t-1}-\mu_{j, l}, \ldots, y_{j, t-d_{j}+1}-\right.$ $\left.\mu_{j, l}\right)^{\mathrm{T}} \in \mathbb{R}^{d_{j}}, \varepsilon_{j, t}$ denotes the sequence of white noise error stimuli, 


$$
G_{j, l}=\left(\begin{array}{ccccc}
\phi_{j, l, 1} & 1 & 0 & \cdots & 0 \\
\phi_{j, l, 2} & 0 & 1 & \cdots & 0 \\
\vdots & \vdots & \vdots & \vdots & \vdots \\
\phi_{j, l, d_{j}-1} & 0 & 0 & \cdots & 1 \\
\phi_{j, l, d_{j}} & 0 & 0 & \cdots & 0
\end{array}\right),
$$

$\phi_{j, l, i}, i=1, \ldots, d_{j}$ and $\theta_{j, l, i}, i=1, \ldots, d_{j}-1$ denote the constant ARMA model coefficients of the $l$-th DLM of the $j$-th output. Associated with the resulting stationary model, the back substitution of the state equation from (10) yields,

$$
\mathcal{Y}_{j, t}=G_{j, l} \mathcal{Y}_{j, t-1}+F_{j, l} \varepsilon_{j, t}=\sum_{i=0}^{\infty}\left(G_{j, l}\right)^{i} F_{j, l} \varepsilon_{j, t-i} .
$$

It should be pointed out that the stationarity condition requires that all the eigenvalues of $G_{j, l}$ are located inside the unit circle and, moreover $\left(G_{j, l}\right)^{i}$ decreases in (11) by increasing $i$ [17], [18]. For justification of these properties refer to [17], [19], [43] for more details.

Remark 4: The nonlinear non-stationary observation forecast at the time instant $k$, i.e. $y_{j, t+k}$ made at the time $t+k-1$ by using the ARMA model based on its linear projection on the last available $p_{j}$ observations $\left\{y_{j, t+k-1}, \ldots, y_{j, t+k-p_{j}}\right\}$, is denoted by $y_{j, t+k}^{\text {lin }}$. This represents a linear approximation to the actual nonlinear observation process. By applying the one-step ahead prediction algorithm (10), $y_{j, t+k}^{\operatorname{lin}_{n}}$ is obtained as,

$$
\begin{aligned}
y_{j, t+k}^{\operatorname{lin}}-\mu_{j, l} & =H\left(G_{j, l} \mathcal{Y}_{j, t+k-1}+F_{j, l} \varepsilon_{j, t+k}\right) \\
& =H\left(\sum_{i=0}^{\infty}\left(G_{j, l}\right)^{i} F_{j, l} \varepsilon_{j, t+k-i}\right) .
\end{aligned}
$$

We denote the $k$-step ahead forecast of $y_{j, t+k}$ that is made at time $t$ based on the linear approximation according to the ARMA model (10) as $\hat{Y}_{j, t+k \mid t}$. The most commonly used criterion for evaluating the performance of the predictor $\hat{Y}_{j, t+k \mid t}$ is considered to be its mean-square error (MSE) from $y_{j, t+k}^{\operatorname{lin}}$ that is defined by $\mathbb{E}\left\{\left(y_{j, t+k}^{\operatorname{lin}}-\hat{Y}_{j, t+k \mid t}\right)^{2}\right\}$. Towards this end, $\hat{Y}_{j, t+k \mid t}$ is obtained by back substitution of (10) into (12) when the noise error terms related to the future time instants are set to zero (i.e., $\varepsilon_{j, t+i}=0$ for $i>0$ ) and by applying (11), as follows

$$
\begin{aligned}
\hat{Y}_{j, t+k \mid t}-\mu_{j, l} & =H\left(\left(G_{j, l}\right)^{k+1} \mathcal{Y}_{j, t-1}+\left(G_{j, l}\right)^{k} F_{j, l} \varepsilon_{j, t}\right) \\
& =H\left(\left(G_{j, l}\right)^{k} \sum_{i=0}^{\infty}\left(G_{j, l}\right)^{i} F_{j, l} \varepsilon_{j, t-i}\right) .
\end{aligned}
$$

The above implies that once the matrix $G_{j, l}$ is determined from the available data within the specified time window, the linear forecast and prediction of the observations can be obtained from (13) for a specific prediction horizon by assuming that the process remains stationary.

Below, in Theorem 1, an upper bound on the mean square error (MSE) of the $k$-step ahead forecasting using the ARMA process with the DLM model formulation is obtained. It should be noted that the overall forecast error has to be computed from the deviation between the predicted observation values, $\hat{Y}_{j, t+k \mid t}$ and their values from the nonlinear non-stationary observation process, $y_{j, t+k}$ as governed by (2). In Theorem 2 stated subsequently, this overall forecast error will be stated as a function of the prediction horizon $(k)$.

First, the following lemma is stated following the results in [44] which is necessary for the proof of our Theorem 1. 
Lemma 1. Let $A$ represent a real square matrix with all its eigenvalues located inside the unit circle, and $B$ represent any real matrix having a proper dimension. Then,

$$
\left\|\sum_{i=0}^{k-1} A^{i} B\left(A^{i}\right)^{\mathrm{T}}\right\| \leq\|B\| \frac{\kappa\left(1-r^{k}\right)}{1-r},
$$

where $\|B\|$ denotes the matrix norm and is defined as the spectral norm $\rho^{1 / 2}\left(B^{\mathrm{T}} B\right), \kappa$ and $r$ are positive constants such that $\rho(A)<r<1$, and $\left\|A^{i}\right\|^{2} \leq \kappa r^{i}$, where $\rho(X)$ denotes the maximum eigenvalue of the matrix $X$.

Proof: The proof of this lemma is provided in Appendix A.

The result in Lemma 1 is now used to obtain an upper bound on the MSE obseryation forecasting error as a function of the prediction horizon $(k)$.

Theorem 1. Consider the ARMA model with the DLM formulation as given by (10). Using this model, the $k$-step ahead forecast of the observation at the time instant $t$, denoted by $\hat{Y}_{j, t+k \mid t}$, has a bounded mean square error of $\mathbb{E}\left\{\left(y_{j, t+k}^{\operatorname{lin}}-\hat{Y}_{j, t+k \mid t}\right)^{2}\right\} \leq \sigma_{\varepsilon_{j}}^{2}\left\|F_{j, l} F_{j, l}^{\mathrm{T}}\right\|\left(\frac{\kappa_{j}\left(1-r_{j}^{k}\right)}{1-r_{j}}\right)$, where $y_{j, t+k}^{\operatorname{lin}}$ is defined according to Remark $4, \sigma_{\varepsilon_{j}}^{2}=\mathbb{E}\left\{\varepsilon_{j, t} \varepsilon_{j, t}\right\}$ denotes the variance of the noise error to $\varepsilon_{j, t}$, and $F_{j, l}$ denotes the vector related to the moving average part of the ARMA model in the DLM formulation, $\kappa_{j}$ and $r_{j}$ denote positive constants such that $\rho\left(G_{j, l}\right)<r_{j}<1$, and for any integer $i>0,\left\|\left(G_{j, l}\right)^{i}\right\|^{2} \leq \kappa_{j} r_{j}^{i}$.

Proof: The proof of this theorem is provided in Appendix A.

It now follows that a threshold for the bound on the forecast error can be chosen by considering it as a percent of the mean $\mu_{j, l}$ within a given window. Hence, one can determine the maximum acceptable value for $k$ in the observation forecasting algorithm for each observation vector (within the time window $(t, t+k])$, by using the $l$-th DLM model, such that $k$ satisfies the condition $\sigma_{\varepsilon_{j}}^{2}\left\|F_{j, l} F_{j, l}^{\mathrm{T}}\right\|\left(\frac{\kappa_{j}\left(1-r_{j}^{k}\right)}{1-r_{j}}\right) \leq \iota \mu_{j, l}$, where $\iota$ denotes the desired percentage of the error in the $k$-step ahead prediction as a percentage of the mean within the window interval $\left(t_{l}-\tau, t_{l}\right]$. We should point out that based on the above criterion for selecting $k$, it can result in different values for each DLM model. Therefore, for ease of notation in the subsequent discussions $k$ is considered to represent the minimum step ahead prediction horizon that satisfies the above mentioned condition for all the DLM models.

Our proposed DLM models based observation forecasting scheme is only capable of forecasting observations as univariate time-series data. In health prediction strategies one is generally more interested in predicting the system hidden states (those that are not necessarily measurable) as well as the system health parameters to evaluate the dynamical system health condition. Our ultimate goal in proposed health monitoring strategy is to utilize the above developed observation forecasting scheme for predicting the propagation of the system states and parameters to future time horizons. Using the $k$-step ahead forecast of the observations, an online prediction method is now developed based on particle filters to predict the evolution of the system states and parameters. In the following subsections, the observation forecasting scheme will be integrated with the particle filters to enhance the prediction performance and capabilities of our proposed methodologies.

\section{Enhancement to Particle Filters for State and Parameter Prediction}

The observation forecasting scheme will now be integrated with our previously developed PF-based dual state and parameter estimation filter, as discussed in Section II-A [13]-[16]. This will allow us to construct a dual estimation algorithm for forecasting and predicting the system states as well as the 


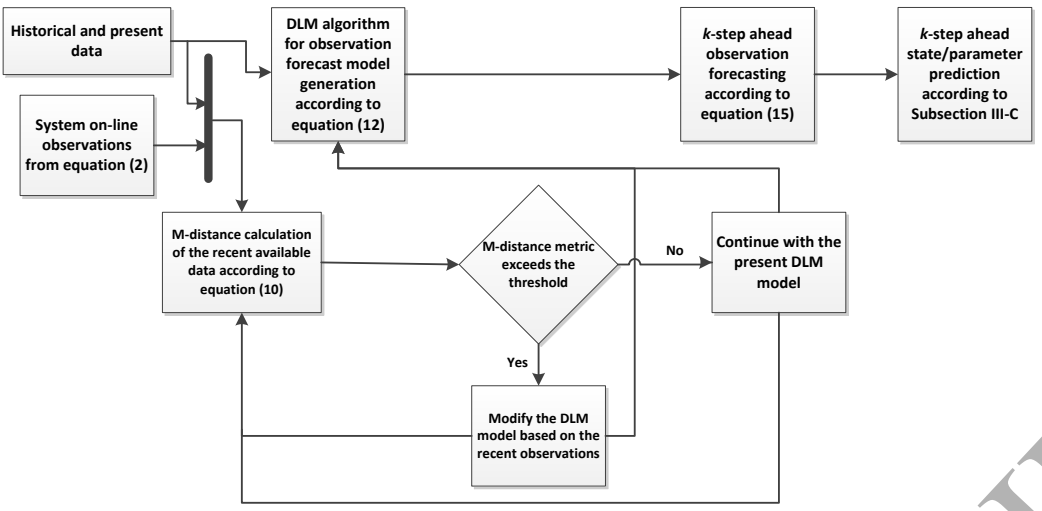

Fig. 2. Flowchart of the health prediction methodology based on the DLM framework.

system slowly time-varying hidden parameters to achieve to a $k$-step ahead horizon. This prediction task is performed by replacing the real observation matrix $y_{t+k}$, where $y_{t+k}=\left(y_{1, t+k}, y_{2, t+k}, \ldots, y_{n_{y}, t+k}\right)^{\mathrm{T}}$ and is also not available after the time instant $t$, by the forecasted observation matrix $\hat{Y}_{t+k \mid t}$, where $\hat{Y}_{t+k \mid t}=\left(\hat{Y}_{1, t+k \mid t}, \hat{Y}_{2, t+k \mid t}, \ldots, \hat{Y}_{n_{y}, t+k \mid t}\right)^{\mathrm{T}}$. Therefore, the resampling algorithm for both state and parameter estimation filters is performed by utilizing the predicted measurements. It should be noted that to differentiate between the forecasted output that is obtained from the fixed-lag DLM forecasting algorithm and the one that is estimated from the particle filters, we use $\hat{Y}_{j, t}$ to designate the forecasted output that is obtained from the DLM-based algorithm, and $\hat{y}_{j, t}$ to designate the estimated output that is obtained from the particle filter algorithm.

Consider the dynamical system (1) and the associated dual state and parameter estimation filtering expressions that are given in Table I for estimation of the a priori and a posteriori state and parameter distributions. By applying the forecasted measurements $\hat{Y}_{t+k \mid t}$, the a priori and a posteriori distributions of the system states and parameters for the $k$-step ahead prediction horizon are approximated accordingly. The flowchart corresponding to our proposed methodology for the system state and health parameters prediction is depicted in Figure 2. In this figure, the update mechanism for the DLM-based observation forecasting module is also provided, which takes advantage of the M-Distance (Mahalanobis distance) metric that is applied to recent observations, to emphasize that the observation forecasting module can be updated at any time that is needed. However, in the last step, the $k$-step ahead state/parameter prediction will be responsible for generating the prediction of the states, parameters, and the measurement outputs $\left(y_{t+k}\right)$ as described in detail in this subsection below.

k-step Ahead State and Parameter Prediction Algorithm: The details corresponding to the states and parameters predictions procedures are provided and summarized below, where $r=1, \ldots, k$, namely,

1) Perform the $r$-step ahead a priori state prediction as follows:

a) Predict the $a$ priori state particles according to $\hat{x}_{t+r \mid t}^{(i)}=f_{t}\left(\hat{x}_{t+r-1 \mid t}^{(i)}, \hat{\theta}_{t+r-1 \mid t}^{\mathrm{T}} \lambda\left(\hat{x}_{t+r-1 \mid t}^{(i)}\right), \omega_{t+r}^{(i)}\right)$, where $\omega_{t+r}^{(i)}$ is the particle noise that is added to the state particle at $t+r$ and is generated from the same distribution as $\omega_{t}^{(i)}$,

b) Approximate the a priori state covariance matrix according to:

$\Sigma_{\hat{x}_{t+r \mid t}}=\frac{1}{N-1} \sum_{i=1}^{N}\left(\hat{x}_{t+r \mid t}^{(i)}-\frac{1}{N} \sum_{j=1}^{N} \hat{x}_{t+r \mid t}^{(j)}\right)\left(\hat{x}_{t+r \mid t}^{(i)}-\frac{1}{N} \sum_{j=1}^{N} \hat{x}_{t+r \mid t}^{(j)}\right)^{\mathrm{T}}$. 
2) Perform the $r$-step ahead a posteriori state prediction as follows:

a) Calculate the state estimation filter weights for resampling as: $\tilde{w}_{x t+r}^{(i)} \triangleq \frac{\rho\left(\hat{Y}_{t+r \mid t} \mid \hat{x}_{t+r \mid t}^{(i)}, \hat{\theta}_{t+r \mid t}\right)}{\sum_{i=1}^{N} \rho\left(\hat{Y}_{t+r \mid t} \mid \hat{x}_{t+r \mid t}^{(i)}, \hat{\theta}_{t+r-1 \mid t}\right)}$,

b) Approximate the a posteriori state distribution from:

$\tilde{\pi}_{x_{t+r \mid t}^{N}}^{N}\left(\mathrm{~d} x_{t+r}\right) \approx \sum_{l=1}^{N_{\mathrm{reg}}} \sum_{i=1}^{N} \tilde{w}_{x_{t+r}}^{(i)} \frac{\left|\mathbb{A}_{t}^{-1}\right|}{b^{n} x} \mathcal{K}\left(\frac{1}{b} \mathbb{A}_{t}^{-1}\left(x_{t+r}^{\mathrm{reg}_{l}}-\hat{x}_{t+r \mid t}^{(i)}\right)\right), \mathbb{A}_{t} \mathbb{A}_{t}^{\mathrm{T}}=\Sigma_{\hat{x}_{t+r \mid t}}$, where the kernel density $\mathcal{K}$ is not the optimal kernel, i.e. the Epanechnikov kernel [45], rather it represents a simple Gaussian kernel,

c) Resample the state estimate particles according to the filter weights $\tilde{w}_{x t+r}^{(i)}$, that is denoted by $\bar{x}_{t+r \mid t}^{(i)}$ and approximate the a posteriori state estimation distribution after resampling according to $\pi_{x_{t+r \mid t}}^{N}\left(\mathrm{~d} x_{t+r}\right)=\frac{1}{N} \sum_{i=1}^{N} \delta_{\bar{x}_{t+r \mid t}^{(i)}}\left(\mathrm{d} x_{t+r}\right)$,

d) Approximate the a posterior state estimation according to $\hat{x}_{t+r \mid t}=\frac{1}{N} \sum_{i=1}^{N} \bar{x}_{t+r \mid t}^{(i)}$.

3) Perform the first step $r$-step ahead a posteriori parameter prediction as follows:

a) Calculate the corrected output prediction error as: $\epsilon_{t+r}\left(\hat{\theta}_{t+r-1 \mid t}^{(j)}\right)=\hat{Y}_{t+r \mid t}-h_{t}\left(\hat{x}_{t+r \mid t}, \hat{\theta}_{t+r-1 \mid t}^{(j)^{\mathrm{T}}} \lambda\left(\hat{x}_{t+r \mid t}\right)\right)$,

b) Calculate $m_{t+r}^{(j)}$ as $m_{t+r}^{(j)}=\hat{\theta}_{t+r-1 \mid t}^{(j)}+\gamma_{t+r} R_{t+r}^{(j)} \psi_{t+r}^{(j)} \epsilon_{t+r}\left(\hat{\theta}_{t+r-1 \mid t}^{(j)}\right)$,

c) Calculate the first step a posteriori parameter prediction by applying the kernel shrinkage algorithm as $\left.\tilde{\theta}_{t+r \mid t}^{(j)}=A m_{t+r}^{(j)}+(I-A) \bar{m}_{t+r-1}+\zeta_{t+r}^{(j)}, \quad \bar{m}_{t+r-1}=\frac{1}{N}\right) \sum_{j=1}^{N} \hat{\theta}_{t+r-1 \mid t}^{(j)}, \quad \zeta_{t+r}^{(j)} \sim \mathcal{N}(0,(I-$ $\left.\left.A^{2}\right) \Sigma_{\hat{\theta}_{t+r-1 \mid t}}\right)$,

4) Perform the second step $r$-step ahead a posteriori parameter prediction as follows:

a) Calculate the parameter estimation filter weights for performing the resampling as follows:

$\tilde{w}_{\theta_{t+r}}^{(j)} \triangleq \frac{\rho\left(\hat{Y}_{t+r|t|} \mid \hat{x}_{t+r \mid t}, \tilde{\theta}_{t+r \mid t}^{(j)}\right)}{\sum_{j=1}^{M} \rho\left(\hat{Y}_{t+r \mid t} \mid \hat{x}_{t+r \mid t}, \tilde{\theta}_{t+r \mid t}^{(j)}\right)}$,

b) Approximate the first step a posteriori parameter distribution according to:

$\tilde{\pi}_{\theta_{t+r \mid t}}^{N}\left(\mathrm{~d} \theta_{t+r}\right) \approx \sum_{j=1}^{N} \tilde{w}_{\theta_{t+r}}^{(j)} \delta_{\tilde{\theta}_{t+r \mid t}^{(j)}}\left(\mathrm{d} \theta_{t+r}\right)$

c) Perform the resampling to approximate the a posteriori parameter prediction distribution based on the resampled parameter predicted particles $\bar{\theta}_{t+r \mid t}^{(j)}$ as: $\pi_{\theta_{t+r \mid t}}^{N}\left(\mathrm{~d} \theta_{t+r}\right)=\frac{1}{N} \sum_{j=1}^{N} \delta_{\bar{\theta}_{t+r \mid t}^{(j)}}\left(\mathrm{d} \theta_{t+r}\right)$

d) Obtain the a posteriori parameter prediction as $\hat{\theta}_{t+r \mid t}=\frac{1}{N} \sum_{j=1}^{N} \bar{\theta}_{t+r \mid t}^{(j)}$,

e) Calculate the a posteriori parameter prediction covariance as $\Sigma_{\hat{\theta}_{t+r \mid t}}=\frac{1}{N-1} \sum_{i=1}^{N}\left(\bar{\theta}_{t+r \mid t}^{(j)}-\hat{\theta}_{t+r \mid t}\right)\left(\bar{\theta}_{t+r \mid t}^{(j)}-\right.$ $\left.\hat{\theta}_{t+r \mid t}\right)^{\mathrm{T}}$.

In view of the above, the state and parameter probability density functions can be generated for the $k$-step ahead prediction by utilizing the predicted observations and by maintaining resampling for the future time instants.

Remark 5: Consider the set $D_{\mathcal{N}}$ as a set for which the functions $f_{t}\left(x_{t}, \theta_{t}^{\mathrm{T}} \lambda\left(x_{t}\right), \omega_{t}\right)$, and $h_{t}\left(x_{t}, \theta_{t}^{\mathrm{T}} \lambda\left(x_{t}\right)\right)$ are sufficiently smooth. Using a projection process as stated in [16], one can ensure that $\hat{\theta}_{t \mid t-1}^{(j)}, j=1, \ldots, N$, and consequently $\hat{\theta}_{t \mid t}^{(j)}$ in the estimation algorithm as stated in Table I will remain inside the subset $\bar{D}$ of $D_{\mathcal{N}}\left(\bar{D} \subset D_{\mathcal{N}}\right)$. On the other hand, for any dynamical system such that $\forall\left(x_{t}, \theta_{t}\right) \in \mathcal{D}_{\mathcal{R}}$, where $\mathcal{D}_{\mathcal{R}}$ denotes the stability region of the dynamical system, the function $h_{t}\left(x_{t}, \theta_{t}^{\mathrm{T}} \lambda\left(x_{t}\right)\right)$ is bounded, where $D_{\mathcal{N}} \subset D_{\mathcal{R}}$. Therefore, the existence of the above mapping in the estimation, and also the $r$-step ahead prediction algorithm for the system states and parameters guarantee that for $r>1, h_{t}\left(\hat{x}_{t+r \mid t+r}, \hat{\theta}_{t+r-1 \mid t+r-1}^{(j)^{\mathrm{T}}} \lambda\left(\hat{x}_{t+r \mid t+r}\right)\right)$ and $h_{t}\left(\hat{x}_{t+r \mid t}, \hat{\theta}_{t+r-1 \mid t}^{(j)^{\mathrm{T}}} \lambda\left(\hat{x}_{t+r \mid t}\right)\right)$ remain bounded where these bounds are denoted by $\mathcal{C}_{1}$ and $\mathcal{C}_{2}$, respectively. 
It should be pointed out that in the parameter estimation filter, the prediction error $\epsilon_{t+r}\left(\hat{\theta}_{t+r-1 \mid t}^{(j)}\right)$ is used to generate a propagation law for the unknown parameters of the system. Due to lack of observations after the time instant $t$, the forecasted observations that utilize the fixed-lag DLM models are used for this purpose. Therefore, as the error increases in the observation forecasting scheme, it can lead to both incorrect predicted parameters and states. Consider the prediction error associated with the $j$-th parameter particle that is calculated at the time instant $t+r$ assuming that observations up to $t+r$ become available as $\epsilon_{t+r}^{(j)}=y_{t+r}-h_{t}\left(\hat{x}_{t+r \mid t+r}, \hat{\theta}_{t+r-1 \mid t+r-1}^{(j)^{\mathrm{T}}} \lambda\left(\hat{x}_{t+r \mid t+r}\right)\right)$, and the $r$-step ahead prediction of $\epsilon_{t+r \mid t}^{(j)}$ assuming that observations up to the time instant $t$ are available as $\epsilon_{t+r \mid t}^{(j)}=\hat{Y}_{t+r \mid t}-h_{t}\left(\hat{x}_{t+r \mid t}, \hat{\theta}_{t+r-1 \mid t}^{(j)^{\mathrm{T}}} \lambda\left(\hat{x}_{t+r \mid t}\right)\right)$. Therefore, the discrepancy between these two quantities will affect the accuracy of the state and parameter prediction algorithm, which is specified according to,

$$
\begin{aligned}
& \left|\epsilon_{t+r}^{(j)}-\epsilon_{t+r \mid t}^{(j)}\right|=\mid y_{t+r}-\hat{Y}_{t+r \mid t}+h_{t}\left(\hat{x}_{t+r \mid t}, \hat{\theta}_{t+r-1 \mid t}^{(j)}\right) \\
& -h_{t}\left(\hat{x}_{t+r \mid t+r}, \hat{\theta}_{t+r-1 \mid t+r-1}^{(j)}\right) \mid \\
& \leq\left|y_{t+r}-\hat{Y}_{t+r \mid t}\right|+\mid h_{t}\left(\hat{x}_{t+r \mid t}, \hat{\theta}_{t+r-1 \mid t}^{(j)}\right) \\
& -h_{t}\left(\hat{x}_{t+r \mid t+r}, \hat{\theta}_{t+r-1 \mid t+r-1}^{(j)}\right) \mid \\
& \leq\left|y_{t+r}-\hat{Y}_{t+r \mid t}\right|+\left|\mathcal{C}_{1}\right| \mathcal{A}\left|\mathcal{C}_{2}\right|
\end{aligned}
$$

where the last inequality is obtained based on the results that are summarized in Remark 4. Now, our main goal here is to investigate the boundedness of $\left|y_{t+r}-\hat{Y}_{t+r \mid t}\right|$ to ensure the boundedness of the prediction algorithm. Towards this end, an element-wise approach for the long-term horizon prediction based on the error that is generated for each forecasted observation that is now compared with the actual nonlinear non-stationary process (2) measurements, is developed in the following theorem.

Theorem 2. Consider the nonlinear stochastic system described by (1) and (2), where the observation noise to $y_{j, t}$ is considered to be generated from a non-stationary stochastic process with bounded variance $\sigma_{y_{j, t}}^{2}$. The $k$-step ahead observation prediction based on the DLM model using the ARMA process followed by the particle filter results in the following bound on the mean square error, that is,

$$
\begin{aligned}
\mathbb{E}\left\{\left(y_{j, t+k}-\hat{Y}_{j, t+k \mid t}\right)^{2}\right\} & \leq \sigma_{y_{j, t}}^{2}+\mathcal{C}_{j, l}+\mathcal{D}_{j, l}+\mathcal{B}_{j, l} \\
& +2 \sqrt{\left(\sigma_{y_{j, t}}^{2}+\mathcal{C}_{j, l}+\mathcal{D}_{j, l}\right) \mathcal{B}_{j, l}}
\end{aligned}
$$

where $\mathcal{B}_{j, l}=\sigma_{\varepsilon_{j}}^{2}\left\|F_{j, l} F_{j, l}^{\mathrm{T}}\right\|\left(\frac{\kappa_{1}\left(1-r_{j}^{k}\right)}{1-r_{j}}\right)$, with $\kappa_{j}$ and $r_{j}, j=1, \ldots, n_{y}$ denote positive constants that satisfy $\left\|G_{j, l}^{i}\right\| \leq \kappa_{j} r_{j}^{i}$. Moreoyer, $\mathcal{C}_{j, l}=h_{j, t+k}^{2}\left(x_{t+k}, \theta_{t+k}^{\mathrm{T}} \lambda\left(x_{t+k}\right)\right)+\mu_{j, l}^{2}-2 \mu_{j, l} h_{j, t+k}\left(x_{t+k}, \theta_{t+k}^{\mathrm{T}} \lambda\left(x_{t+k}\right)\right)$ and $\mathcal{D}_{j, l}=$ $\sigma_{\varepsilon_{j}}^{2}\left\|F_{j, l} F_{j, l}^{\mathrm{T}}\right\|\left(\frac{\kappa_{j}}{1-r_{j}}\right)$.

Proof: The proof of this theorem is provided in Appendix A.

We would like to point out that a more complex time-series forecasting scheme that is based on neural networks can be used to implement this part of our methodology (refer to our recent work in [46]).

\section{REMAining USEFUl Life (RUL) EVALUATION}

The system model that is defined in (1) and (2) is suitable for model-based prognosis specifically in the case that the system health parameters (that are denoted by $\lambda\left(x_{t}\right)$ ) are affected by degradation damage through the fault vector $\theta_{t}$. By performing a system health tracking and its evolution prediction to the future, the remaining useful life (RUL) of the system can be evaluated by taking into account the probability of the failure distribution. This is accomplished according to a known criterion on the 
maximum acceptable change (critical bound) of the system health parameters. In implementation of the health tracking step, the filtering method presented in Section II-A is used. The developed and proposed prediction algorithm that is presented in Section III is also utilized for implementing the health prediction step.

It follows from Theorem 2 that as the prediction horizon is extended, the parameter prediction errors do increase accordingly. For evaluating the performance of our prediction scheme in terms of changes in the system health parameters, given that the true values of the parameters are assumed to be unknown, the percentage root mean square error (PRMSE) criterion is used for the estimated outputs instead of the parameters. This is computed according to [29] as follows, $\operatorname{PRMSE}_{y_{j}}(i)=100 \sqrt{\frac{1}{M} \sum_{j=t_{1}}^{j=t_{M}}\left(\frac{\hat{y}_{j, m+i}-y_{j, m+i}}{y_{j, m+i}}\right)^{2}}$, where $\hat{y}_{j, m+i}$ denotes the predicted value of the $j$-th system output from the particle filters at the time $m+i$ where $m$ denotes the time instants at which the DLM model is updated, $m \in\left\{t_{l_{1}}, \ldots, t_{l_{M}}\right\}$, and $M$ refers to the total number of the DLM models in the entire observation trajectory, $y_{j, m+i}$ denotes the actual measured output (observation) at the time $m+i$, and the mean is taken over all $m+i, m=t_{l_{1}}, \ldots, t_{l_{M}}$, time instants in the prediction horizon, with $i=1, \ldots, k$. The number of steps ahead parameters prediction is chosen from the outputs $\mathrm{PRMSE}_{y_{j}}$ results based on the considered acceptable threshold for each output. It should be emphasized that the prediction error $\epsilon_{t+i}$, which is used in the state and parameter prediction algorithm is calculated as the difference between the forecasted observation and the predicted observation from the particle filter after estimating the states and parameters as $\epsilon_{t+i}=\hat{Y}_{t+i \mid t}-\hat{y}_{t+i}$. Therefore, to evaluate the performance of the prediction scheme for states and parameters, $\hat{y}_{t+i}=h\left(\hat{x}_{t+i \mid t}, \hat{\theta}_{t+i-1 \mid t}^{\mathrm{T}} \lambda\left(\hat{x}_{t+i \mid t}\right)\right)$ is utilized which also incorporates the error due to the state and parameter prediction algorithm.

To calculate the RUL of the system when multiple health parameters are estimated, we first denote and select $\theta_{\mathrm{cr}}^{(s)}, s=1, \ldots, n_{\theta}$, as the critical value for the $s$-th health parameter. The following rule is now utilized for evaluating the RUL at the time $t$ [47], namely $\operatorname{RUL}(t)=t_{j}-t$, where $t_{j}$ is defined as $t_{j}=\min _{t_{j}}\left\{\hat{\theta}_{t_{j}}^{(s)}-\theta_{\mathrm{cr}}^{(s)} \geq 0, s=1, \ldots, n_{\theta}\right\}$, i.e. the time at which the first parameter that is associated with the specific degradation damage reaches its critical value. Once the critical value is reached in one of the parameters, the maintenance action must be performed. Choosing a suitable value for $\theta_{\mathrm{cr}}^{(s)}$ is application specific and is generally determined based on the system performance and operator experience. In most applications and problems selecting an exact value for the RUL is not feasible or desirable. Therefore, an acceptable bound is considered as a confidence interval for the RUL prediction. The above procedure is to estimate the RUL at most $k$-steps before the occurrence of a total failure. As one gets closer to the failure time, the RUL is approximated more accurately due to readjustments in the prediction scheme based on more recent and reliable observations.

In the following section computational complexity of our developed algorithm for the dual state and parameter estimation and their propagation prediction is quantitatively determined and evaluated. The complexity results are compared with the ones that correspond to conventional augmented parameter and state estimation methods as developed in [5], [48] based on particle filters having fixed weights and equally weighted propagation law for particles for a long-term prediction horizon.

\section{COMPlexity AnAlysis}

In this section, the computational complexity of our proposed prediction scheme based on our previously developed dual state and parameter estimation method [14]-[16] that is integrated with the fixed-lag DLM model observation forecasting scheme for long-term prediction of the system states and parameters is quan- 
TABLE II

DEFINITIONS AND DIMENSIONS OF THE ENTITIES IN THE DUAL STATE AND PARAMETER ESTIMATION SCHEME.

\begin{tabular}{|c|c|c|}
\hline Variable & Dimension & Definition \\
\hline$x_{t}$ & $\mathbb{R}^{n_{x}}$ & state vector \\
$\theta_{t}$ & $\mathbb{R}^{n_{\theta}}$ & parameter vector \\
$\Sigma_{x}$ & $\mathbb{R}^{n_{x} \times n_{x}}$ & state estimate covariance matrix \\
$P_{t}$ & $\mathbb{Z}^{+}$ & adaptive step size \\
$A$ & $\mathbb{R}^{n_{\theta} \times n_{\theta}}$ & shrinkage matrix \\
$\Sigma_{\theta}$ & $\mathbb{R}^{n_{\theta} \times n_{\theta}}$ & parameter estimate covariance matrix \\
$W_{t}$ & $\mathbb{R}^{n_{x} \times n_{x}}$ & process noise variance \\
$V_{\theta_{t}}$ & $\mathbb{R}^{n_{\theta} \times n_{\theta}}$ & parameter estimate distribution variance \\
$y_{t}$ & $\mathbb{R}^{n_{y}}$ & measurement output \\
$f_{t}\left(x_{t}, \theta_{t}^{\mathrm{T}} \lambda\left(x_{t}\right), \omega_{t}\right)$ & $\mathbb{R}^{n_{x} \times 1}$ & state dynamic function \\
$h_{t}\left(x_{t}, \theta_{t}^{\mathrm{T}} \lambda\left(x_{t}\right)\right)$ & $\mathbb{R}^{n_{y} \times 1}$ & observation function \\
\hline
\end{tabular}

titatively obtained and analyzed. The analysis is based on the number of floating-point operations (flops) that are required for any given algorithm. A flop is defined as one addition, subtraction, multiplication, or division of two floating-point numbers. However, there are certain procedures where their complexity cannot be measured by using flops, for example for generating random numbers and for evaluating a nonlinear function.

On the other hand, the relationship between flop complexity and time complexity in execution of an algorithm depends on many other factors that are not necessarily reflected in the flop complexity measure. Therefore, in this paper the EF complexity that is introduced in [30] for an operation will be utilized for conducting the complexity analysis. In the EF complexity, the number of flops that result in the same computational time as a given operation is evaluated through the so-called proportionality coefficients. Consequently, one can determine how the computational time will increase as the problem size increases. The EF metric is mostly evaluated for those operations that depend on matrix and vector manipulations.

The dimensions and definitions of some of the entities that are used in the EF analysis of our proposed PF-based state and parameter estimation algorithm are provided in Table II. Given the computational complexity of some common matrix manipulations that are shown in Table III, the EP complexity of our proposed scheme is now summarized in Table IV for the state estimation/prediction step and in Table $\mathrm{V}$ for the parameter estimation/prediction step along with the observation forecasting scheme. The EP quantities in these two tables correspond to only one iteration of the scheme as explained in Subsection III-C. In our proposed algorithm instead of using $y_{t}$ in each prediction iteration, the forecasted observation matrix $\hat{Y}_{t}$ is utilized. The distribution of the system states and outputs are also considered as Gaussian (the same as the parameters distribution) according to [30].

The coefficients $c_{1}, c_{2}, c_{3}$ and $c_{4}$ are used to represent the complexity of random number generation, resampling, regularization, and ARMA model, respectively, since their complexities cannot be measured by using flops. Therefore, their complexities have to be estimated by analyzing the actual computational time that is consumed by various segments of the algorithm [30]. It should be noted that Gaussian likelihood calculations are also included in the resampling step. The Schur decomposition is also used in the process of covariance matrix decomposition to generate the new particle populations in the filters. The complexity of the above operation increases cubically as the dimension of the problem increases. The complexity related to initialization step is ignored in the tables since initialization of state and parameter estimation filters are performed only once and in the start of the algorithm execution.

The complexity of our proposed dual state and parameter estimation and its propagation predictions can 
TABLE III

COMPUTATIONAL COMPLEXITY OF CERTAIN COMMON MATRIX OPERATIONS.

\begin{tabular}{|c|c|c|c|}
\hline Operation & Matrix size & Multiplication & Addition \\
\hline$A+A$ & $A \in \mathbb{R}^{n \times m}$ & - & $n m$ \\
$A . B$ & $A \in \mathbb{R}^{n \times m}, B \in \mathbb{R}^{m \times l}$ & lmn & $(m-1) \ln$ \\
$B . C$ & $B \in \mathbb{R}^{m \times n}, C \in \mathbb{R}^{n \times 1}$ & $n m$ & $(n-1) m$ \\
$D^{-1}$ & $D \in \mathbb{R}^{n \times n}$ & $n^{3}$ & - \\
\hline
\end{tabular}

TABLE IV

THE EQUIVALENT COMPLEXITY OF THE STATE ESTIMATION/PREDICTION MODULE.

\begin{tabular}{|c|c|c|c|c|}
\hline Instruction & Mult. & Add & Func. Eval. & Other \\
\hline$\left[U_{1}, T_{1}\right]=\operatorname{schur}\left(\sum_{\hat{x}_{t+r-1 \mid t}}\right)$ & - & - & & $10 n_{x}^{3}$ \\
\hline$R_{1}=\operatorname{randn}\left(n_{x}, N\right)$ & - & - & & $N n_{x} c_{1}$ \\
\hline$\omega_{t+r}^{(i)}=\left(U_{1} \sqrt{T_{1}}\right) R_{1}$ & $n_{x}^{3}+N n_{x}^{2}$ & $\left(n_{x}-1\right) n_{x}^{2}+N\left(n_{x}\right.$ & & $n_{x}^{2}$ \\
\hline$\hat{x}_{t+r \mid t}^{(i)}=f_{t}\left(\hat{x}_{t+r-1 \mid t}^{(i)}, \hat{\theta}_{t+r-1 \mid t}^{\mathrm{T}} \lambda\left(\hat{x}_{t+r-1 \mid t}^{(i)}\right), \omega_{t+r}^{(i)}\right)$ & - & - & & - \\
\hline$\hat{y}_{t+r \mid t}^{(i)}=h_{t}\left(\hat{x}_{t+r \mid t}^{(i)}, \hat{\theta}_{t+r-1 \mid t}^{\mathrm{T}} \lambda\left(x_{t+r \mid t}^{(i)}\right)\right)$ & - & - & & - \\
\hline$\Sigma_{\hat{x}_{t+r \mid t}}=\frac{1}{N-1} \sum_{i=1}^{N}\left(\bar{x}_{t+r \mid t}^{(i)}-\hat{x}_{t+r \mid t}\right)\left(\bar{x}_{t+r \mid t}^{(i)}-\hat{x}_{t+r \mid t}\right)^{\mathrm{T}}$ & $N n_{x}^{2}$ & $2 N n_{x}$ & & - \\
\hline Regularization and resampling to find weights $w_{x_{t+r}}^{(i)}$ and $\bar{x}_{t+r \mid t}^{(i)}$ & - & - & & $N n_{x} c_{2}+N n_{x} c_{3}$ \\
\hline$\hat{x}_{t+r \mid t}=\frac{1}{N} \sum_{i=1}^{N} \bar{x}_{t+r \mid t}^{(i)}$ & $n_{x}$ & 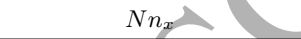 & - & - \\
\hline Total & $n_{x}^{3}+2 N n_{x}^{2}+n_{x}$ & $n_{x}^{3}+(N-1) n_{x}^{2}+2 N n_{x}$ & $N\left(n_{x}+n_{y}\right)$ & $\begin{array}{c}10 n_{x}^{3}+n_{x}^{2} \\
N n_{x}\left(c_{1}+c_{2}+c_{3}\right)\end{array}$ \\
\hline
\end{tabular}

be compared with the complexity of a standard/conventional algorithm ( [5], [48]) for state and constant parameter estimation using particle filters when the particles are propagated with fixed weights to the future time instants [5], [48] as stated in Table VI. In the standard/conventional PF-based prediction algorithm ( [5], [48]) the parameters are augmented to the state vector, therefore the dimension of the augmented state and parameter model becomes $n_{x}+n_{\theta}$, and the complexity associated with the resampling step, i.e., $c_{2}$ was removed from the EF complexity evaluation.

In Table VII, the EF complexity of the two methods are summarized. To compare the EF complexity

TABLE V

THE EQUIVALENT COMPLEXITY OF THE PARAMETER ESTIMATION/PREDICTION MODULE USING THE OBSERVATION PREDICTION SCHEME.

\begin{tabular}{|c|c|c|c|c|}
\hline Instruction & Mult. & Add & Func. Eval. & Other \\
\hline $\bar{y}_{t+r \mid t}^{(j)}=h_{t}\left(\hat{x}_{t+r \mid t}, \theta_{t+r-1 \mid t}^{(j)^{\mathrm{T}}} \lambda\left(\hat{x}_{t+r \mid t}\right)\right)$ & - & - & $N n_{y}$ & - \\
\hline$\Sigma_{\theta}=\left(I-A^{2}\right) \Sigma_{\hat{\theta}_{t+r-1 \mid t}}$ & $n_{\theta}^{3}$ & $\left(n_{\theta}-1\right) n_{\theta}^{2}+n_{\theta}^{2}$ & - & - \\
\hline$\epsilon_{t+r}^{(j)}=\hat{Y}_{t+r}-\bar{y}_{t+r \mid t}^{(j)}$ & - & $N n_{y}$ & - & - \\
\hline$(j)=\frac{d h}{d \theta}$ & - & - & $n_{y} n_{\theta}$ & - \\
\hline$P_{t+r}^{(j)}=\gamma_{t+r}\left(\sqrt{\operatorname{trace}\left(\epsilon_{t+r}^{(j)} \epsilon_{t+r}^{(j)^{\mathrm{T}}}\right.}\right)$ & $N+N n_{y}$ & $N\left(n_{y}-1\right)+N n_{y}$ & - & - \\
\hline$\left[U_{2}, L_{2}\right]=\operatorname{schur}\left(\Sigma_{\theta}\right)$ & - & - & - & $10 n_{\theta}^{3}$ \\
\hline$\left.R_{2}=\operatorname{randn}\left(n_{\theta}, N\right)\right)$ & - & - & - & $N n_{\theta} c_{1}$ \\
\hline$\zeta_{t+r}^{(j)}=\left(U_{2} \sqrt{L_{2}}\right) R_{2}$ & $n_{\theta}^{3}+N n_{\theta}^{2}$ & $\left(n_{\theta}-1\right) n_{\theta}^{2}+N\left(n_{\theta}-1\right) n_{\theta}$ & $n_{\theta}^{2}$ & - \\
\hline$m_{t+r}^{(j)}=\hat{\theta}_{t+r-1 \mid t}^{(j)}+P_{t+r}^{(j)} \psi_{t+r}^{(j)} \epsilon_{t+r}^{(j)}$ & $N\left(n_{y} n_{\theta}+n_{\theta}\right)$ & $N\left(n_{y}-1\right) n_{\theta}+N n_{\theta}$ & - & - \\
\hline$\tilde{\theta}_{t+r \mid t}^{(j)}=A m_{t+r}^{(j)}+(I-A) \frac{1}{N} \sum_{j=1}^{N} \hat{\theta}_{t+r-1 \mid t}^{(j)}+\zeta_{t+r}^{(j)}$ & $N n_{\theta}^{2}+n_{\theta}$ & $N n_{\theta}^{2}+2 N n_{\theta}+N n_{\theta}+n_{\theta}^{2}$ & - & - \\
\hline$\hat{y}_{t+r \mid t}=h_{t}\left(\hat{x}_{t+r \mid t}, \hat{\theta}_{t+r \mid t}^{(j)^{\mathrm{T}}} \lambda\left(\hat{x}_{t+r \mid t}\right)\right)$ & - & - & $N n_{y}$ & - \\
\hline Resampling to find weights, $w_{\theta_{t+r}}^{(j)}$, and $\bar{\theta}_{t+r \mid t}^{(j)}$ & - & - & - & $N n_{\theta} c_{2}$ \\
\hline$\hat{\theta}_{t+r \mid t}=\frac{1}{N} \sum_{j=1}^{N} \bar{\theta}_{t+r \mid t}^{(j)}$ & $n_{\theta}$ & $N n_{\theta}$ & - & - \\
\hline$\Sigma_{\hat{\theta}_{t+r \mid t}}=\frac{1}{N-1} \sum_{i=1}^{N}\left(\bar{\theta}_{t+r \mid t}^{(j)}-\hat{\theta}_{t+r \mid t}\right)\left(\bar{\theta}_{t+r \mid t}^{(j)}-\hat{\theta}_{t+r \mid t}\right)^{\mathrm{T}}$ & $N n_{\theta}^{2}$ & $2 N n_{\theta}$ & - & - \\
\hline M-Distance calculation for $s ́$ observations & - & - & - & $\dot{s}\left(q^{-1}\right) n_{y}$ \\
\hline ARMA evaluation after $\tau$ observations & - & - & - & $\tau c_{4} n_{y}$ \\
\hline Total & $\begin{array}{c}2 n_{\theta}^{3}+3 N n_{\theta}^{2} \\
+(N+2) n_{\theta}+N n_{\theta} n_{y} \\
+N\left(n_{y}+1\right)\end{array}$ & $\begin{array}{c}2 n_{\theta}^{3}+2 N n_{\theta}^{2} \\
+5 N n_{\theta}+3 N n_{y}-N \\
+N n_{\theta} n_{y}\end{array}$ & $\begin{array}{c}n_{\theta}^{2}+2 N n_{y} \\
\quad+n_{y} n_{\theta}\end{array}$ & $\begin{array}{c}10 n_{\theta}^{3}+N n_{\theta} c_{1}+N n_{\theta} c_{2} \\
+\dot{s}(\dot{q})^{-1} n_{y} \\
+\tau c_{4} n_{y}\end{array}$ \\
\hline
\end{tabular}


TABLE VI

THE EQUIVALENT COMPLEXITY OF THE AUGMENTED STATE AND PARAMETER ESTIMATION/PREDICTION SCHEME.

\begin{tabular}{|c|c|c|c|c|}
\hline Instruction & Mult. & Add & Func. Eval. & Other \\
\hline$\left[U_{1}, T_{1}\right]=\operatorname{schur}\left(\Sigma_{x, \theta}\right)$ & - & - & - & $10\left(n_{x}+n_{\theta}\right)^{3}$ \\
\hline$R_{1}=\operatorname{randn}\left(n_{x}+n_{\theta}, N\right)$ & - & - & - & $N\left(n_{x}+n_{\theta}\right) c_{1}$ \\
\hline$\omega_{t+r}^{(i)}=\left(U_{1} \sqrt{T_{1}}\right) R_{1}$ & $\left(n_{x}+n_{\theta}\right)^{3}+N\left(n_{x}+n_{\theta}\right)^{2}$ & $\left(n_{x}+n_{\theta}-1\right)\left(n_{x}+n_{\theta}\right)^{2}$ & - & $\left(n_{x}+n_{\theta}\right)^{2}$ \\
\hline & & $+N\left(n_{x}+n_{\theta}-1\right)\left(n_{x}+n_{\theta}\right)$ & & \\
\hline$\omega_{x_{t+r}}^{(i)}=\omega_{t+r}^{(i)}\left(1: n_{x}\right)$ & - & - & - & - \\
\hline $\begin{array}{c}\omega_{\theta_{t+r}}=\left(I-A^{2}\right) \omega_{t+r}^{(i)}\left(n_{x}+1: n_{x}+n_{\theta}\right) \\
\text { state/parameter augmentation: }\left[\hat{x}_{t+r \mid t}^{(i)} ; \hat{\theta}_{t+r \mid t}^{(i)}\right]=\end{array}$ & $n_{\theta}^{3}$ & $\left(n_{\theta}-1\right) n_{\theta}^{2}+n_{\theta}^{2}$ & - & - \\
\hline$\left[f_{t}\left(\hat{x}_{t+r-1 \mid t}^{(i)}, \hat{\theta}_{t+r-1 \mid t}^{(i)^{\mathrm{T}}} \lambda\left(\hat{x}_{t+r-1 \mid t}^{(i)}\right), \omega_{x_{t+r}}^{(i)}\right) ; \hat{\theta}_{t+r-1 \mid t}^{(i)}\right]$ & - & - & $N\left(n_{x}+n_{\theta}\right)$ & - \\
\hline$\hat{y}_{t+r \mid t}^{(i)}=h_{t}\left(\hat{x}_{t+r \mid t}^{(i)}, \hat{\theta}_{t+r \mid t}^{(i)^{\mathrm{T}}} \lambda\left(\hat{x}_{t+r \mid t}^{(i)}\right)\right)$ & - & - & $\mathrm{Nr}$ & - \\
\hline 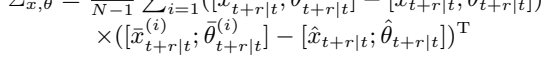 & $N\left(n_{x}+n_{\theta}\right)^{2}$ & $2 N\left(n_{x}+n_{\theta}\right)$ & & - \\
\hline $\begin{array}{c}\text { Regularization without resampling } \\
\text { to find, } \bar{x}_{t+r \mid t}^{(i)} \text { and } \bar{\theta}_{t+r \mid t}^{(i)} \\
{\left[\hat{x}_{t+r \mid t} ; \hat{\theta}_{t+r \mid t}\right]=\left[\frac{1}{N} \sum_{i=1}^{N} \bar{x}_{t+r \mid t}^{(i)} ; \frac{1}{N} \sum_{i=1}^{N} \bar{\theta}_{t+r \mid t}^{(i)}\right]}\end{array}$ & $\begin{array}{c}- \\
n_{x}+n_{\theta} \\
\end{array}$ & $\begin{array}{c}- \\
N\left(n_{x}+n_{\theta}\right)\end{array}$ & & $\begin{array}{c}N\left(n_{x}+n_{\theta}\right) c_{3} \\
-\end{array}$ \\
\hline Total & $\begin{array}{c}2 n_{\theta}^{3}+n_{x}^{3}+n_{x}^{2}\left(2 N+3 n_{\theta}\right) \\
+n_{\theta}^{2}\left(3 n_{x}+2 N\right)+4 N n_{\theta} n_{x} \\
+n_{x}+n_{\theta}\end{array}$ & $\begin{aligned} n_{x}^{3}+ & 2 n_{\theta}^{3}+n_{x}^{2}\left(3 n_{\theta}-1+N\right) \\
+ & n_{\theta}^{2}\left(N-1+3 n_{x}\right) \\
& +n_{x} n_{\theta}(2 N-2)\end{aligned}$ & $\begin{array}{l}N\left(n_{x}+n_{\theta}\right) \\
+N n_{y}\end{array}$ & $\begin{array}{c}10 n_{x}^{3}+10 n_{\theta}^{3} \\
n_{x}^{2}\left(30 n_{\theta}+1\right) \\
+n_{\theta}^{2}\left(30 n_{x}+1\right) \\
+n_{x}\left(N c_{1}+2 n_{\theta}+N c_{3}\right) \\
+n_{\theta}\left(N c_{1}+N c_{3}\right)\end{array}$ \\
\hline
\end{tabular}

TABLE VII

THE TOTAL EQUIVALENT COMPLEXITY OF THE FILTERS.

\begin{tabular}{|c|c|}
\hline Prediction Method & Total Equivalent Complexity \\
\hline $\begin{array}{l}\text { DLM-Based Prediction Method } \\
\text { (this paper) }\end{array}$ & $\begin{array}{c}C_{D L M}\left(n_{x}, n_{\theta}, c_{1}, c_{2}, c_{3}, c_{4}, N\right)=12 n_{x}^{3}+14 n_{\theta}^{3}+N\left(3 n_{x}^{2}+5 n_{\theta}^{2}+6 n_{\theta}+2 n_{\theta} n_{y}+7 n_{y}+3 n_{x}+c_{1}\left(n_{x}+n_{\theta}\right)+\right. \\
\left.c_{2}\left(n_{x}+n_{\theta}\right)+c_{3} n_{x}\right) n_{x}+n_{\theta}^{2}+2 n_{\theta}+n_{y} n_{\theta}+n_{y}\left(\tau c_{4}+(\dot{q})^{-1} \dot{s}\right)\end{array}$ \\
\hline $\begin{array}{l}\text { Standard PF-Based Prediction Method } \\
\text { ( [5], [48]) }\end{array}$ & $\begin{array}{c}C_{P F}\left(n_{x}, n_{\theta}, c_{1}, c_{3}, N\right)=12 n_{x}^{3}+14 n_{\theta}^{3}+N\left(3 n_{x}^{2}+3 n_{\theta}^{2}+6 n_{x} n_{\theta}+\left(1+c_{1}+c_{3}\right) n_{x}+\left(1+c_{1}+c_{3}\right) n_{\theta}+n_{y}\right) \\
+36 n_{\theta}^{2} n_{x}+36 n_{x}^{2} n_{\theta}+n_{x}+n_{\theta}\end{array}$ \\
\hline
\end{tabular}

results, only the dominant parts of $C_{D L M}\left(n_{x}, n_{\theta}, c_{1}, c_{2}, c_{3}, c_{4}, N\right)$ (that represent the EF complexity of our proposed method) and $C_{P F}\left(n_{x}, n_{\theta}, c_{1}, c_{3}, N\right)$ (that represent the conventional state and parameter prediction method based on particle filters in the literature [5], [48]) are provided. This selection is justified by the fact that $N \gg 1$, therefore the dominant parts are the ones that are related to $N$.

Let us assume that the time interval $\tau=\beta N$, and $\beta<1$ is a constant. To quantitatively evaluate the EF complexity, two cases are considered now. In the first case, it is assumed that the measurement dimension, $n_{y}$ as well as the parameter dimension $\left(n_{\theta} \leq n_{y}\right)$ is much smaller than the state dimension $\left(n_{x}\right)$. Consequently, the components that are related to only $n_{x}$ are considered, that is

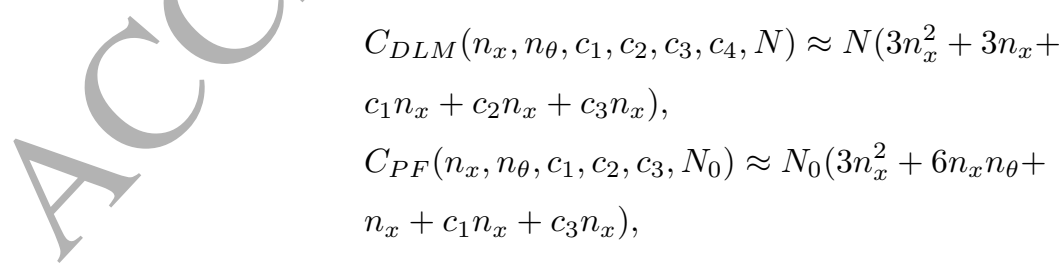

where $N_{0}$ denotes the number of particles that are required for implementation of the conventional methods [5], [48].

It follows from (16) that for achieving the same EF complexity in the two methods, the number of particles that are required in our proposed method can be determined based on the number of the particles that are required in the conventional methods [5], [48] as follows,

$$
N=N_{0}\left(1-\frac{2 n_{x}+c_{2} n_{x}-6 n_{x} n_{\theta}}{3 n_{x}^{2}+3 n_{x}+c_{1} n_{x}+c_{2} n_{x}+c_{3} n_{x}}\right),
$$


It should be pointed out that the resampling algorithm deals with ordering, therefore it is assumed that in the worst case its computational complexity is much greater than other operations in the algorithm. Hence, it follows clearly that for a given complexity, since $c_{2}+2>6 n_{\theta}$ (this results in $N<N_{0}$ ), one should use fewer particles in our proposed method as compared to the conventional methods [5], [48]. Therefore, the conventional method can be implemented with more particles as compared to our proposed method to achieve the same complexity, whereas increasing the particles in our proposed method results in more computational complexity and consequently more implementation cost. To decrease the cost of implementing our proposed algorithm, the coefficient of $N_{0}$ in (17) has to be as much as possible near to 1 . Consequently, one should get

$$
3 n_{x}^{2}+3 n_{x}+c_{1} n_{x}+c_{2} n_{x}+c_{3} n_{x} \gg 2 n_{x}+c_{2} n_{x}-6 n_{x} n_{\theta} .
$$

The above inequality is always satisfied since the left-hand side term in (18) is much greater than the right-hand side term due to existence of terms corresponding to the EF complexity of the random number generation $\left(c_{1}\right)$ and regularization $\left(c_{3}\right)$. The most interesting result in this case is that the choice of resampling algorithm does not affect the inequality that is stated in (18).

In the second case, it is assumed that the measurement dimension $n_{y}$ and the state dimension $n_{x}$ are larger than the dimension of the parameters $n_{\theta}$, i.e. $n_{x}, n_{y} \gg n_{\theta}$. Hence, to evaluate the EF complexity of the two methods (according to Table VII), the dominant terms are selected as the ones that are functions of $N, n_{x}$ and/or $n_{y}$, while the terms that are dependent on only $N$ and $n_{\theta}$ are ignored. Therefore, the EF complexity evaluations in this case become, $C_{D L M}\left(n_{x}, n_{\theta}, c_{1}, c_{2}, c_{3}, c_{4}, N\right) \approx N\left(3 n_{x}^{2}+2 n_{\theta} n_{y}+7 n_{y}+\right.$ $\left.3 n_{x}+c_{1} n_{x}+c_{2} n_{x}+c_{3} n_{x}+n_{y} \beta c_{4}\right)$ and $C_{P F}\left(n_{x}, n_{\theta}, c_{1}, c_{2}, c_{3}, N_{0}\right) \approx N_{0}\left(3 n_{x}^{2}+6 n_{x} n_{\theta}+\left(1+c_{1}+c_{3}\right) n_{x}+n_{y}\right)$. Finally, from the above expressions it follows that to achieve the same EF complexity for the two methods, the number of particles that are required in our proposed method is determined based on the number of particles that are required in the conventional methods [5], [48] (the number of particles is set to $N_{0}$ ) and is set to $N=N_{0}\left(1-\frac{c_{2} n_{x}+6 n_{y}+2 n_{x}+2 n_{\theta} n_{y}+n_{y} \beta c_{4}-6 n_{\theta} n_{x}}{3 n_{x}^{2}+2 n_{\theta} n_{y}+\left(3+c_{1}+c_{2}+c_{3}\right) n_{x}+7 n_{y}+n_{y} \beta c_{4}}\right)$.

By applying the same analysis as in the first case, to achieve less cost in the implementation of our proposed algorithm as compared to the conventional methods, one must have

$$
\begin{aligned}
3 n_{x}^{2}+2 n_{\theta} n_{y} 4 & \left(3+c_{1}+c_{2}+c_{3}\right) n_{x}+7 n_{y}+n_{y} \beta c_{4} \gg c_{2} n_{x} \\
+ & 6 n_{y}+2 n_{x}+2 n_{\theta} n_{y}+n_{y} \beta c_{4}-6 n_{\theta} n_{x} .
\end{aligned}
$$

Let us assume that $n_{x}, n_{y} \gg n_{\theta}$, so that the above inequality can be simplified as follows

$$
3 n_{x}^{2}+n_{x}+c_{1} n_{x}+c_{3} n_{x}+7 n_{y} \gg 6 n_{y}-6 n_{x} n_{\theta}
$$

where the above condition is always satisfied due to the high EF complexity of $c_{1}$ and $c_{3}$ and the fact that the evaluation of our proposed algorithm implementation cost is independent of the choice of $c_{2}$ and $c_{4}$ (the ARMA model calculation). In simulation results that are presented in Section VI, it will be shown that under this circumstance where one employs the same number of particles in the two methods, the computational time (which is equivalent to the EF complexity) of our method when the ARMA structure with variable order is used (for implementing the observation forecasting scheme), would be comparable to and in some cases actually significantly less than the conventional methods having invariant particle weights. 


\section{Vi. Failure Prognosis of a Gas Turbine Engine}

The application of our proposed PF-based prediction method for health monitoring and prognosis of a gas turbine engine is presented in this section. Our approach is applied and implemented for failure prognosis of the engine, when the system is assumed to be affected by health degradation phenomena. Our proposed and developed prediction scheme is demonstrated and illustrated to be capable of handling cases when non-Gaussian process noise is applied to the system. Moreover, the performance of our proposed state and parameter prediction scheme is evaluated and investigated under general scenarios of degradation in both the turbine and the compressor components due to erosion and fouling phenomena.

\section{A. Model Overview}

The mathematical model of a gas turbine that is used in this paper is a single spool jet engine that was developed in [31]-[33]. The four engine states are the combustion chamber pressure and temperature, $P_{\mathrm{CC}}$ and $T_{\mathrm{CC}}$, respectively, the spool speed $S$, and the nozzle outlet pressure $P_{\mathrm{NLT}}$. The continuous-time state space model of the system is given as follows,

$$
\begin{aligned}
& \dot{T}_{\mathrm{CC}}=\frac{1}{c_{\mathrm{v}} \dot{m}_{\mathrm{cc}}}\left[\left(c_{\mathrm{p}} T_{\mathrm{C}} \theta_{m_{\mathrm{C}}} \dot{m}_{\mathrm{C}}+\eta_{\mathrm{CC}} H_{\mathrm{u}} \dot{m}_{\mathrm{f}}-\hat{c}_{\mathrm{p}} T_{\mathrm{CC}} \theta_{m_{\mathrm{T}}} \dot{m}_{\mathrm{T}}\right)\right. \\
& \left.-c_{\mathrm{v}} T_{\mathrm{CC}}\left(\theta_{m_{\mathrm{C}}} \dot{m}_{\mathrm{C}}+\dot{m}_{\mathrm{f}}-\theta_{m_{\mathrm{T}}} \dot{m}_{\mathrm{T}}\right)\right]+\omega_{1} \\
& \dot{S}=\frac{\eta_{\text {mech }} \theta_{m_{\mathrm{T}}} \dot{m}_{\mathrm{T}} c_{\mathrm{p}}\left(T_{\mathrm{CC}}-T_{\mathrm{T}}\right)-\theta_{m_{\mathrm{C}}} \dot{m}_{\mathrm{C}} c_{\mathrm{p}}\left(T_{\mathrm{C}}-\left(T_{\mathrm{d}}+\omega_{2}\right)\right)}{\mathrm{J} S\left(\frac{\pi}{30}\right)^{2}}, \\
& \dot{P}_{\mathrm{CC}}=\frac{P_{\mathrm{CC}}}{T_{\mathrm{CC}}} \frac{1}{c_{\mathrm{v}} \dot{m}_{\mathrm{cc}}}\left[\left(c_{\mathrm{p}} T_{\mathrm{C}} \theta_{m_{\mathrm{C}}} \dot{m}_{\mathrm{C}}+\eta_{\mathrm{CC}} H_{\mathrm{u}} \dot{m}_{\mathrm{f}}\right.\right. \\
& \left.\left.-c_{\mathrm{p}} T_{\mathrm{CC}} \theta_{m_{\mathrm{T}}} \dot{m}_{\mathrm{T}}\right)-c_{\mathrm{V}} T_{\mathrm{CC}}\left(\theta_{m_{\mathrm{C}}} \dot{m}_{\mathrm{C}}+\dot{m}_{\mathrm{f}}-\theta_{m_{\mathrm{T}}} \dot{m}_{\mathrm{T}}\right)\right] \\
& +\frac{\gamma \mathrm{R} T_{\mathrm{CC}}}{V_{\mathrm{CC}}}\left(\theta_{m_{\mathrm{C}}} \dot{m}_{\mathrm{C}}+\dot{m}_{\mathrm{f}}-\theta_{m_{\mathrm{T}}} \dot{m}_{\mathrm{T}}\right)+\omega_{3}, \\
& \dot{P}_{\mathrm{NLT}}=\frac{T_{\mathrm{M}}}{V_{\mathrm{M}}}\left(\theta_{m_{\mathrm{T}}} \dot{m}_{\mathrm{T}}+\frac{\beta}{\beta+1} \theta_{m_{\mathrm{C}}} \dot{m}_{\mathrm{C}}-\left(\dot{m}_{\text {Nozzle }}+\omega_{4}\right)\right)
\end{aligned}
$$

where the physical significance of all the model parameters is provided in Table VIII (also refer to [31]-[33], and $\omega_{i}, i=1, \ldots, 4$ denote the process noise that are applied to the system dynamics.

The five gas turbine measured outputs are considered to be the compressor temperature $\left(y_{1}\right)$, the combustion chamber pressure $\left(y_{2}\right)$, the spool speed $\left(y_{3}\right)$, the nozzle outlet pressure $\left(y_{4}\right)$, and the turbine temperature $\left(y_{5}\right)$, namely

$$
\begin{aligned}
y_{1} & =T_{\mathrm{C}}=T_{\text {diffuser }}\left[1+\frac{1}{\theta_{\eta_{\mathrm{C}}} \eta_{\mathrm{C}}}\left[\left(\frac{P_{\mathrm{CC}}}{P_{\text {diffuzer }}}\right)^{\frac{\gamma-1}{\gamma}}-1\right]\right]+\nu_{1}, \\
y_{2} & =P_{\mathrm{CC}}+\nu_{2}, y_{3}=S+\nu_{3}, y_{4}=P_{\mathrm{NLT}}+\nu_{4}, \\
y_{5} & =T_{\mathrm{CC}}\left[1-\theta_{\eta_{\mathrm{T}}} \eta_{\mathrm{T}}\left(1-\left(\frac{P_{\mathrm{NLT}}}{P_{\mathrm{CC}}}\right)^{\frac{\gamma-1}{\gamma}}\right]+\nu_{5} .\right.
\end{aligned}
$$

It should be noted that all the measurements are noise corrupted where the noise are denoted by $\nu_{i}, i=1, \ldots, 5$. The specific noise levels are taken from [32], where the standard deviations are taken as percentage of the nominal values at a typical cruise condition. Specifically, we set the standard deviations as follows: $\sigma_{y_{1}}=0.280, \sigma_{y_{2}}=0.164, \sigma_{y_{3}}=0.051, \sigma_{y_{4}}=0.164$, and $\sigma_{y_{5}}=0.097$. 
TABLE VIII

MOdel PARAMETERS Description.

\begin{tabular}{|c||c|c||c|}
\hline parameter & description & parameter & description \\
\hline \hline$c_{\mathrm{V}}$ & Specific heat at constant pressure, $\frac{\mathrm{J}}{\mathrm{kg} \cdot \mathrm{K}}$ & $T_{\mathrm{T}}$ & Turbine temperature, $\mathrm{K}$ \\
$c_{\mathrm{p}}$ & Specific heat at constant volume, $\frac{\mathrm{J}}{\mathrm{kg} \cdot \mathrm{K}}$ & $T_{\mathrm{d}}$ & Intake temperature, $\mathrm{K}$ \\
$\dot{m}_{\mathrm{cc}}$ & Combustion chamber mass flow rate, $\mathrm{kg} / \mathrm{s}$ & $\mathrm{J}$ & Rotor moment of inertia, $\mathrm{kg} \cdot \mathrm{m}^{2}$ \\
$T_{\mathrm{C}}$ & Compressor temperature, $\mathrm{K}$ & $\mathrm{R}$ & Gas constant, $\frac{\mathrm{J}}{\mathrm{kg} \cdot \mathrm{K}}$ \\
$H_{\mathrm{u}}$ & Fuel specific heat, $\frac{\mathrm{J}}{\mathrm{kg}}$ & $\gamma$ & Heat capacity ratio \\
$\eta_{\mathrm{CC}}$ & Combustion chamber efficiency & $V_{\mathrm{CC}}$ & Combustion camber Volume, $\mathrm{m}^{3}$ \\
$\dot{m}_{\mathrm{f}}$ & Fuel flow, kg/s & $T_{\mathrm{M}}$ & Mixer temperature, $\mathrm{K}$ \\
$\dot{m}_{\mathrm{T}}$ & Turbine mass flow rate, $\mathrm{kg} / \mathrm{s}$ & $V_{\mathrm{M}}$ & Mixer volume, $\mathrm{m}^{3}$ \\
$\eta_{\mathrm{T}}$ & Turbine efficiency & $\dot{m}_{\text {nozzle }}$ & Nozzle mass flow rate,, $\mathrm{kg} / \mathrm{s}$ \\
$\dot{m}_{\mathrm{C}}$ & Compressor mass flow rate, $\mathrm{kg} / \mathrm{s}$ & $P_{\text {diffuzer }}$ & Diffuzer pressure, bar \\
$\eta_{\mathrm{C}}$ & Compressor efficiency & $T_{\text {diffuzer }}$ & Diffuzer temperature, $\mathrm{K}$ \\
$\eta_{\mathrm{mech}}$ & Spool mechanical efficiency & & \\
\hline
\end{tabular}

To discretize the above continuous-time model for implementation of our proposed dual state and parameter estimation and prediction particle filters, a simple Euler Backward method is applied with the sampling period of $T_{s}=10$ msec.

The system health parameters are represented by the compressor and the turbine efficiency, $\eta_{\mathrm{C}}$ and $\eta_{\mathrm{T}}$, respectively, and the compressor and turbine mass flow capacities, $\dot{m}_{\mathrm{C}}$ and $\dot{m}_{\mathrm{T}}$, respectively. A fault vector is incorporated in the model (19) to represent the effects of the system health parameters that are denoted by $\theta=\left[\theta_{\eta_{\mathrm{C}}}, \theta_{m_{\mathrm{C}}}, \theta_{\eta_{\mathrm{T}}}, \theta_{m_{\mathrm{T}}}\right]^{\mathrm{T}}$. Each parameter variation is a manifestation of changes in the fault vector and is considered as a multiplicative fault type.

\section{B. Simulation Scenarios}

In [14]-[16], capabilities of the developed state and parameter estimation algorithm were shown in case of abrupt degradation damages that are modeled as multiplicative faults vector. In the present scenarios considered here the engine is assumed to be subjected to degradation damages that are due to the compressor fouling and turbine erosion that cause gradual drifts in the system health parameters. A slowly changing linear degradation model is applied to the compressor health parameters during 1000 cycles of the aircraft engine operation that cause a $3 \%$ drop in the compressor efficiency and $1.5 \%$ drop in its mass flow capacity, followed by a recovery through washing after 1000 cycles.

A cycle refers to a single ground-air-ground (GAG) flight cycle [49], where the recorded cruise data related to each flight cycle is used for prognosis of the gas turbine engine due to gradual degradations. The erosion degradation in the turbine is propagated through a quadratic evolution during the entire 1500 cycles of simulations that cause a $6 \%$ drop in the turbine efficiency and a $3 \%$ increase in its mass flow capacity. It should be noted that fouling and erosion degradation phenomena follow linear propagation patterns under low degradation index values, however as the degradation index increases they would not necessarily follow a linear profile [50]. It is assumed that the flight data are generated from the same engine thrust in the cruise mode of the aircraft operation.

In order to determine the input fuel flow to maintain the same level of thrust in all the flight cycles, a simple PID controller has been implemented that takes the error between the desired fixed engine pressure ratio (which is used as an indicator for the engine thrust that is not measurable) and the actual pressure ratio in each flight cycle when the engine is affected by the fouling and/or the erosion degradations. 
TABLE IX

PSEUDO CODE OF OUR PROPOSED PREDICTION SCHEME.

1) Collect 150 available observations.

2) For $j=1: n_{y}$

a) Construct the input-output database for the ARMA model,

b) Use the recent 150 data points for the DLM model construction,

c) Find the best DLM structure according to the ARMA model having the maximum order ARMA $(4,1)$ that fits $y_{j}$, by using the AIC (Akaike Information Criterion) criterion [39]. In case of a constant model order, a fixed order model structure ARMA $(4,1)$ is selected in this step,

d) Apply the RLS to obtain the coefficients of the ARMA model (DLM model parameters) recursively for the selected data set,

e) Predict the behavior of $y_{j}$ for the next 60 -steps ahead $\left(\hat{Y}_{j, t+k \mid t}, k=1, \ldots, 60\right)$ using the approximated DLM model.

3) Run the PF algorithm by applying the forecasted observation vector $\hat{Y}_{t+k \mid t}$, to obtain the $k$-step ahead prediction of states and parameters.

4) Move the observation window for the minimum $s \geq 10$ of the recently observed data points.

a) Calculate the M-distance for the recently received $s$ observations and the last available 35 data points in the time window of 150 data points (used for the DLM model construction),

b) If the M-distance exceeds the threshold, go to Step 2 and re-calculate the DLM parameters,

c) If the M-distance does not exceed the threshold, continue with the previously constructed DLM model for the 60-steps ahead prediction of the observations and go to Step 3.

A moving window of 150 observation data is used with $N=150$ particles, where the window is moved according to the previously described Mahalanobis distance (M-distance) criterion after each batch of 10 observations become available $(s=10)$. We use the available 150 observation data points $(\tau=150)^{1}$ in the observation forecasting scheme to develop the fixed-lag DLM model and the last 35 data points for validation of the DLM model $(s=35)$. The size of the sliding window in the M-distance algorithm is chosen as $\dot{q}=10$, and the threshold in the change detection algorithm is set to $\delta=1.5$. The parameters of the ARMA models related to each observation time window are adjusted based on the RLS method. The order of the time-varying ARMA structure is considered to be variable. For the AR part of the ARMA model the number of delays varies from 1 to 4 , while the order of the MA part is fixed and set to 1 . The criterion for choosing the order of the ARMA model in each time window for generating the DLM model is devised based on the Akaike information criterion or AIC [39]. To compare the variable order time-varying ARMA model results to forecast the system observations with the fixed order ARMA model, an ARMA structure with the order of 4, i.e., AR(4) and MA(1) is also considered. The pseudo code of the proposed prediction algorithm is provided in Table IX.

In the remainder of this section, the outline of the simulation case studies and objectives that are described and proyided below is first presented. The RUL results for the aircraft gas turbine engine are provided subject to degradations due to fouling and erosion separately. These results are evaluated comparatively using three methods, namely the variable order model (VOM), the fixed order model (FOM), and the W/O RS schemes. The performance of the RUL prediction results are also evaluated subject to changes and various selections of the DLM parameters. Finally, we have performed a quantitative analysis and comparisons on the time complexity of the proposed prediction schemes to illustrate and justify the merits of our proposed methodologies as compared to other methods that are available in the literature.

\footnotetext{
${ }^{1}$ The minimum number of data points needed for convergence of the RLS algorithm for estimation of the DLM model parameters, for this application, is found to be 150 data points.
} 


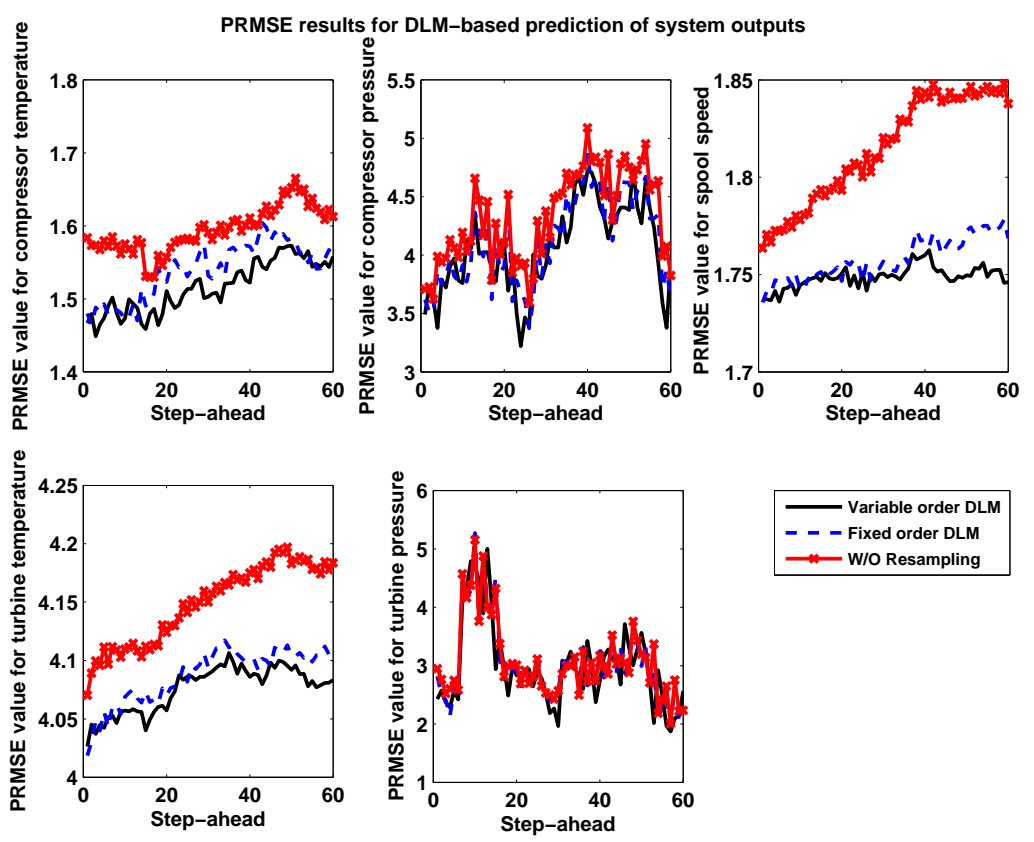

Fig. 3. PRMSE values of the predicted outputs using the Fixed Order Model (FOM), the Variable Order Model (VOM) and the model without resampling (W/O RS).

To demonstrate the effectiveness of our proposed prediction algorithm when compared to the developed prediction algorithm with the constant weights in the particle filter based estimation method in the literature [5], [48], we evaluate the $\operatorname{PRMSE}_{y_{j}}(i)$ for $i=1, \ldots, 60$, and $j=1, \ldots, 5$ corresponding to three methods. Specifically, the three methods considered are (a) the DLM-based particle filter prediction with constant model order for the observation forecasting, (b) the variable DLM model order, and finally (c) the particle filter based prediction with equally weighted particles without resampling ( [5], [48]) are presented in Table $\mathrm{X}$ and in Figure 3 depicting the predicted observations.

From the results that are obtained it can be concluded that our developed DLM-based particle filter prediction algorithm (FOM) and (VOM) for prediction of the system states and health parameters outperform the conventional PF-based prediction method ( [5], [48]) in the sense of PRMSE $y_{j}$ values on the predicted observations. This is clearly indicated when the prediction horizon is extended to 60-steps ahead. Note that the $\mathrm{PRMSE}_{y_{j}}$ results corresponding to the compressor and the turbine pressures are almost the same for all the three methods.

The maximum step-ahead prediction horizon is chosen based on the mean PRMSE $_{y_{j}}$ (percentage of the mean square error for the measurement outputs) as stated in Table $\mathrm{X}$, whereas beyond the chosen horizon $k=60$ the error becomes unacceptable due to the deviation of $\mathbb{E}\left\{\left(y_{j, t+k}-\hat{Y}_{j, t+k \mid t}\right)^{2}\right\}$ from $\iota \mu_{j, l}, \iota=0.01$. The PRMSE analysis is conducted based on 48 different DLM models which are generated throughout the entire set of scenarios.

In the next subsection, the obtained parameter estimates are used to evaluate the remaining useful life (RUL) of the gas turbine engine under both fouling and erosion degradation scenarios. 

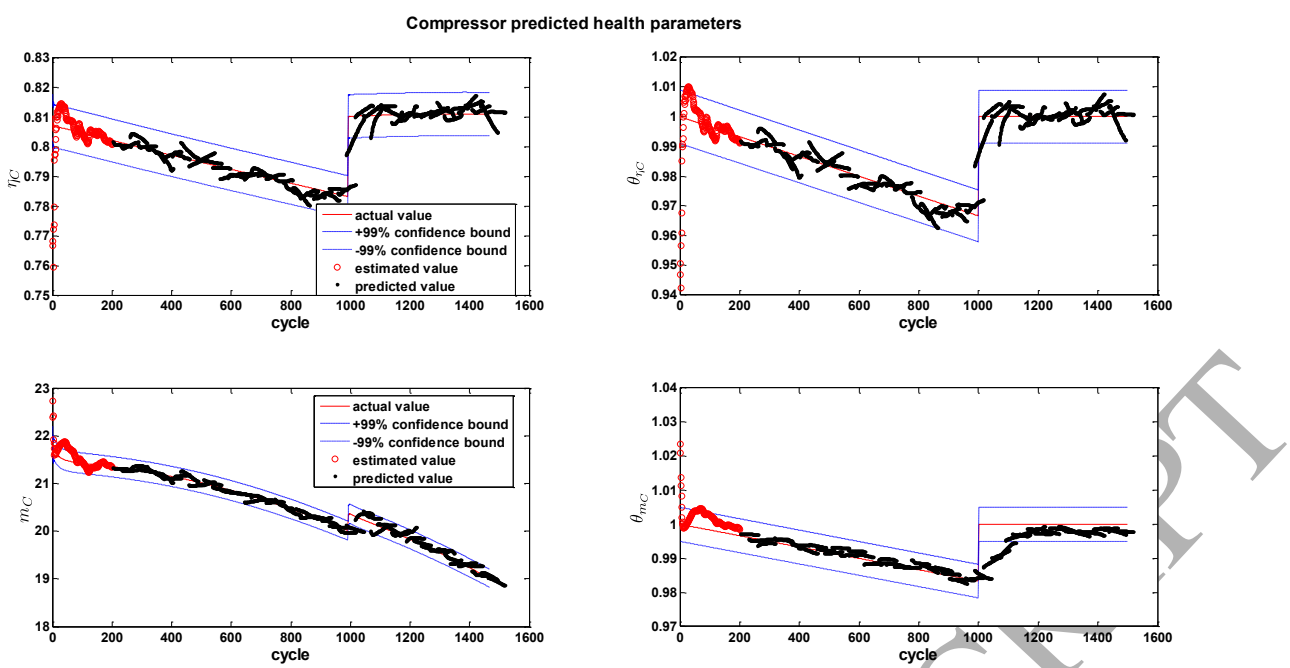

Fig. 4. The predicted compressor health parameters and their related fault vectors.

TABLE X

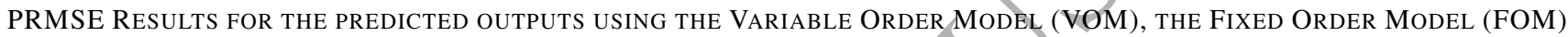
AND THE METHOD WITHOUT RESAMPLING (W/O RS).

\begin{tabular}{|c|c|c|c||c|c|c|}
\hline \multirow{2}{*}{ Output } & \multicolumn{3}{|c||}{ Max PRMSE } & \multicolumn{3}{c|}{ Mean PRMSE } \\
\cline { 2 - 7 } & VOM & FOM & W/ORS & VOM & FOM & $W / O R S$ \\
\hline$T_{\mathrm{C}}$ & 1.5729 & 1.6043 & 1.6653 & 1.5160 & 1.5387 & 1.5946 \\
$P_{\mathrm{CC}}$ & 4.7397 & 4.8860 & 5.0903 & 4.0564 & 4.1217 & 4.3287 \\
$N$ & 1.7625 & 1.7782 & 1.8481 & 1.7487 & 1.7577 & 1.8147 \\
$P_{\mathrm{NLT}}$ & 5.0001 & 5.2708 & 5.1541 & 2.9853 & 3.0272 & 3.0720 \\
$T_{\mathrm{T}}$ & 4.1064 & 4.1173 & 4.1971 & 4.0757 & 4.0872 & 4.1488 \\
\hline
\end{tabular}

\section{RUL Prediction}

For evaluating the RUL of the gas turbine engine, as per the criteria stated in Section V, all the four parameters are considered for health evaluation and for estimating the system RUL in a prediction horizon of 60-steps ahead. The RUL is evaluated within the prediction windows that start from two subsequent flight cycles 854 and 877 (these two windows are corresponding to two updated DLM models) that include the ground truth failure cycle in their 60 -steps ahead prediction horizon. The ground truth failure cycle for the compressor health evaluation due to the fouling phenomenon is located at the cycle 900 . For the turbine health evaluation, the windows starting from the cycles 1114 and 1138 are considered (this is due to the same reason as stated for the compressor failure time windows) while the ground truth failure cycle due to the erosion is located at the cycle 1162. The predicted health parameters and their fault parameters along with the actual degradations are depicted in Figures 4 and 5 for the compressor and the turbine, respectively.

The $\pm 1 \%$ confidence intervals for the compressor efficiency and the mass flow capacity indicate that the two predicted parameters are located within the confidence intervals for most of the time in simulations. For the turbine parameters, these confidence intervals are found to be $\pm 1 \%$ for the turbine efficiency and $\pm 2 \%$ for the turbine mass flow capacity. It should be noted that the first 150 data points in the Figures 4 and 5 are corresponding to the estimated health parameters (one-step ahead prediction) that are calculated 

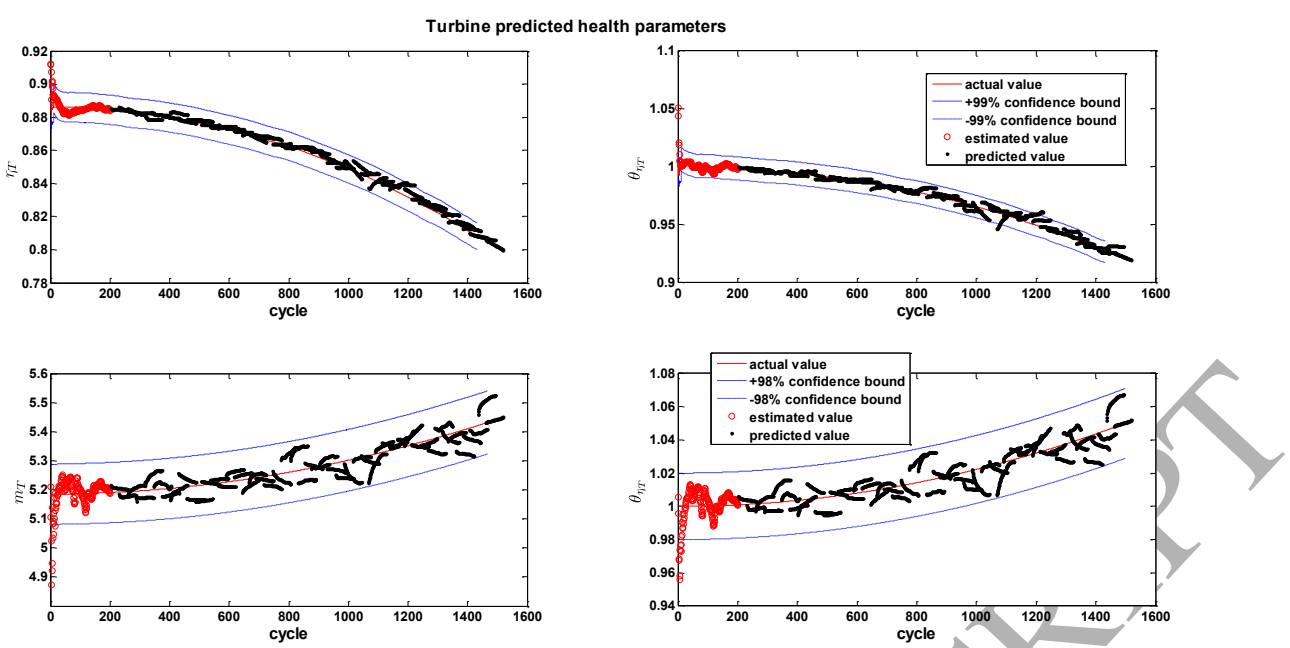

Fig. 5. The predicted turbine health parameters and their related fault vectors.

in presence of the system observations. As stated earlier, as new observations become available the $k$ step ahead prediction of the system states and parameters will be performed based on recently received observations. Therefore, as far as each prediction window for the health parameters in Figures 4 and 5 are concerned there are 150 data points of estimated values that are only shown for the first prediction window and they are removed from the figures for the remainder of the prediction windows to obtain clearer figures for distinguishing the predicted parameters from the estimated ones.

The critical values for the parameter degradations are considered to be a $3 \%$ decrease in the compressor efficiency [50] along with a $1.5 \%$ decrease in the mass flow capacity due to the fouling phenomenon. This implies that after achieving this level of deficiency in any of these parameters, the compressor must be taken for a wash up. On the other hand, the critical values for detecting erosion in the gas turbine are considered to be a $6 \%$ decrease in the turbine efficiency and a $3 \%$ increase in the mass flow capacity.

Fouling scenario: Corresponding to the obtained compressor health parameters prediction, two subsequent time windows are considered. These time windows start from the flight cycles 854 and 877 , respectively. For determining the flight cycle at which the maximum probability of failure has occurred, a probabilistic method is proposed. In this method the distribution of the predicted data related to the system health parameters (i.e., in the compressor mass flow capacity and efficiency in the fouling scenario and the turbine mass flow capacity and efficiency in the erosion scenario) in each time window of 60-steps ahead horizon is fitted to a Gaussian distribution. Consequently, the amount of changes in the mean of this distribution through consequent prediction windows determine changes in its related health parameter. Consider the health indicator vector for the fouling scenario as $\mathrm{HI}_{t+1: t+k}=\left(\begin{array}{lll}\hat{\theta}_{\eta_{\mathrm{C} t+1 \mid t}} & \cdots & \hat{\theta}_{\eta_{\mathrm{C} t+k \mid t}} \\ \hat{\theta}_{m_{\mathrm{C} t+1 \mid t}} & \cdots & \hat{\theta}_{m_{\mathrm{C} t+k \mid t}}\end{array}\right)$, where the mean value is given by mean $\left(\mathrm{HI}_{\mathrm{t}+1: \mathrm{t}+\mathrm{k}}\right)=\left(\mu_{\eta_{\mathrm{C}}}, \mu_{m_{\mathrm{C}}}\right)^{\mathrm{T}}$, and the variance in denoted by $\operatorname{var}\left(\mathrm{HI}_{\mathrm{t}+1: \mathrm{t}+\mathrm{k}}\right)=\left(\begin{array}{cc}\delta_{\theta_{\eta_{\mathrm{C}}}}^{2} & 0 \\ 0 & \delta_{\theta_{m_{\mathrm{C}}}}^{2}\end{array}\right)$. Hence, the Gaussian probability function corresponding to each health 
indicator (the compressor health parameters) is governed by

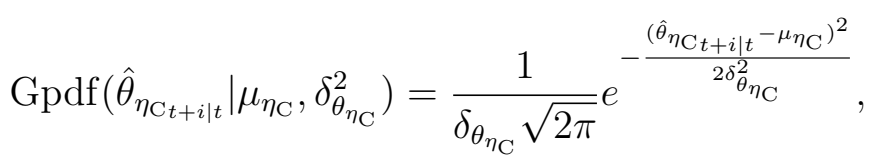

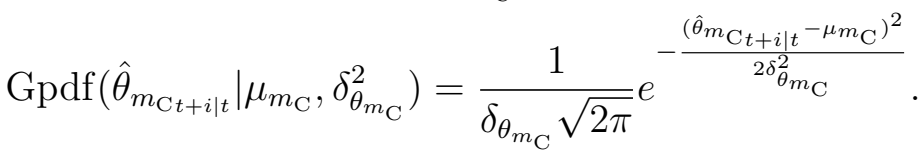

Moreover, the failure cycle corresponding to each health indicator is obtained as the cycle at which the predicted health parameter exceeds the $99.5 \%$ confidence bound around the critical value of that health parameter. Therefore, considering that the fouling phenomenon is identified by its decreasing effect on both the compressor health parameters, the upper bound on the critical values of the compressor health parameters is considered as the criterion for determining the system failure cycle due to fouling. Due to the fact that the failure cycle according to this rule can be more than one cycle, the one corresponding to the maximum probability density function is considered as the failure cycle, given the assumed health parameter which is obtained as follows

$$
\begin{gathered}
\text { FailureCycle }_{\eta_{\mathrm{C}}}=t+j \text {, such that } \hat{\theta}_{\eta_{\mathrm{C} t+j \mid t}} \leq(1.05) \theta_{\eta_{\mathrm{C}}}^{\mathrm{cr}} \\
\text { and } t+j=\operatorname{argmax}_{j=1}^{k} \operatorname{Gpdf}\left(\hat{\theta}_{\eta_{\mathrm{C} t+j \mid t}}\right), \\
\text { FailureCycle }_{m_{\mathrm{C}}}=t+l \text {, such that } \hat{\theta}_{m_{\mathrm{C} t+l \mid t}} \leq(1.05) \theta_{m_{\mathrm{C}}}^{\text {cr }} \\
\text { and } t+l=\operatorname{argmax}_{l=1}^{k} \operatorname{Gpdf}\left(\hat{\theta}_{m_{\mathrm{C} t+l \mid t}}\right),
\end{gathered}
$$

where $\theta_{\eta_{\mathrm{C}}}^{\mathrm{cr}}$ and $\theta_{m_{\mathrm{C}}}^{\mathrm{cr}}$ denote the compressor efficiency and the mass flow capacity critical values, respectively. Finally, the failure cycle for the system is determined as the minimum of FailureCycle $\eta_{\mathrm{C}}$ and FailureCycle $_{m_{\mathrm{C}}}$ as FailureCycle $=\min \left(\right.$ FailureCycle $_{\eta_{\mathrm{C}}}$, FailureCycle $\left._{m_{\mathrm{C}}}\right)$.

By performing a probabilistic study on the distribution of the predicted data in the considered two time windows, the results that are summarized in Table XI and Figures 6 and 7 are obtained for the prediction performed from all the three methods. The ground truth failure cycle, which is the flight cycle 900 , corresponds to $\theta_{\eta_{\mathrm{C}}}^{\text {cr }}=0.97$ and $\theta_{m_{\mathrm{C}}}^{\text {cr }}=0.985$.

In Table XI, the mean and standard deviation of the distribution for the 60 -steps ahead predicted data (when they are fitted to a Gaussian distribution), are shown which are based on the results that are depicted in Figures 6 and 7. In these figures the changes in compressor fault parameters distributions for the two considered time windows that are close to the failure cycle are presented for the three prediction methods. In addition to the distributions that are related to changes in the health parameter fault vector, the cycles at which the calculated probabilities are achieved are also plotted in Figure 6 for the compressor efficiency and in Figure 7 for the compressor mass flow capacity. In cases that these distributions are reliable Gaussian distributions, the failure cycle can be predicted as the cycle at which the probability distribution reaches its maximum value.

The results presented in Table XI guarantee that as one gets closer to the failure cycle, the distributions means related to prediction of both compressor health parameter faults are located within the $99.5 \%$ confidence bound around the actual critical values. However, the results corresponding to the conventional method (W/O RS) does not follow the correct direction for the degradation propagation. While the distribution mean for the predicted values is located around 0.9674 in the first time window, as the window moves towards closer points around the failure cycle, the distribution mean increases to 0.9705 , which 
TABLE XI

PREDICTED DISTRIBUTIONS DUE TO THE COMPRESSOR FOULING.

\begin{tabular}{|c|c|c|c||c|c||c|}
\hline \multirow{2}{*}{ Parameter } & \multicolumn{2}{|c|}{ Prediction from cycle 854} & \multicolumn{2}{c||}{ Prediction from cycle 877} & \multirow{2}{*}{ within $\pm 99.5 \%$ of critical value } \\
\cline { 2 - 6 } & method & mean & \pm std & mean & \pm std & yes \\
\hline$\theta_{\eta_{C}}$ & VOM & 0.967 & 0.0003 & 0.967 & 0.0009 & yes \\
& FOM & 0.966 & 0.0006 & 0.967 & 0.0017 & yes \\
& W/O RS & 0.967 & 0.0028 & 0.970 & 0.0009 & yes \\
\hline$\theta_{m_{C}}$ & VOM & 0.986 & 0.0004 & 0.985 & 0.0001 & yes \\
& FOM & 0.985 & 0.0004 & 0.985 & 0.0001 & yes \\
& W/O RS & 0.984 & 0.0018 & 0.982 & 0.0002 & \\
\hline
\end{tabular}

is not correct due to the fact that the fouling phenomenon causes gradual degradation in the compressor health parameters that have decreasing effects on the compressor health parameters (not decreasing in one window and increasing in the consequent one). Therefore, the RUL prediction for the compressor fouling scenario using this method cannot give us a reliable result in terms of the failure cycle. As one can observe from the results presented in Figure 6, the maximum probability of failure is achieved at several cycles (instead of one cycle). Considering the probability distributions around the related flight cycles as presented in Figure 6, the failure cycle can be predicted from both windows for the VOM and the FOM, whereas for the W/O RS only from the first time window one can obtain a reliable prediction for the failure cycle.

Similar to the compressor efficiency fault vector results, the corresponding results for the compressor mass flow capacity are also located in the $99.5 \%$ confidence bound around the actual critical value. Moreover, for predicting the failure cycle, following the results presented in Figure 7 one can use the distributions that are related to the VOM from both windows for failure cycle prediction. However, the W/O RS is not suitable for failure cycle prediction from the first window due to unreliable probability distribution in this time window. This causes several cycles with maximum probability of failure values, and the FOM is not suitable for failure cycle prediction from the second window for the same reason.

Finally, the predicted RUL from the starting point of the two considered time windows is calculated as the difference between the start cycle in the considered time window and the predicted failure cycle in that window for each health parameter separately as shown in Table XII. The predicted failure cycle is obtained as the cycle at which the maximum probability distribution in the related time window is reached (for both fault vectors of the compressor and the turbine). In the first time window starting at 854 the actual RUL is $46(900-854=46)$, and in the second time window starting at 877 the actual RUL is $23(900-877=23)$. The RUL error is also specified in Table XII, which is calculated as the difference between the actual RUL and the predicted RUL from the different methods.

In view of the results in this table, it is concluded that the VOM is capable of predicting the RUL from two time windows for both compressor health parameters and as the time window moves towards the actual failure cycle, the RUL prediction becomes more accurate such that in the second time window the RUL can be predicted within \pm 5 cycles around the actual RUL. Moreover, the FOM method can determine the RUL based on both health parameters in the first time window within \pm 14 cycles around the actual RUL, whereas in the second time window only one of the parameters can be used for the RUL prediction. However, the prediction method based on the W/O RS does not have sufficient accuracy for the RUL prediction based on both health parameters of the system from the two time windows. The criterion 

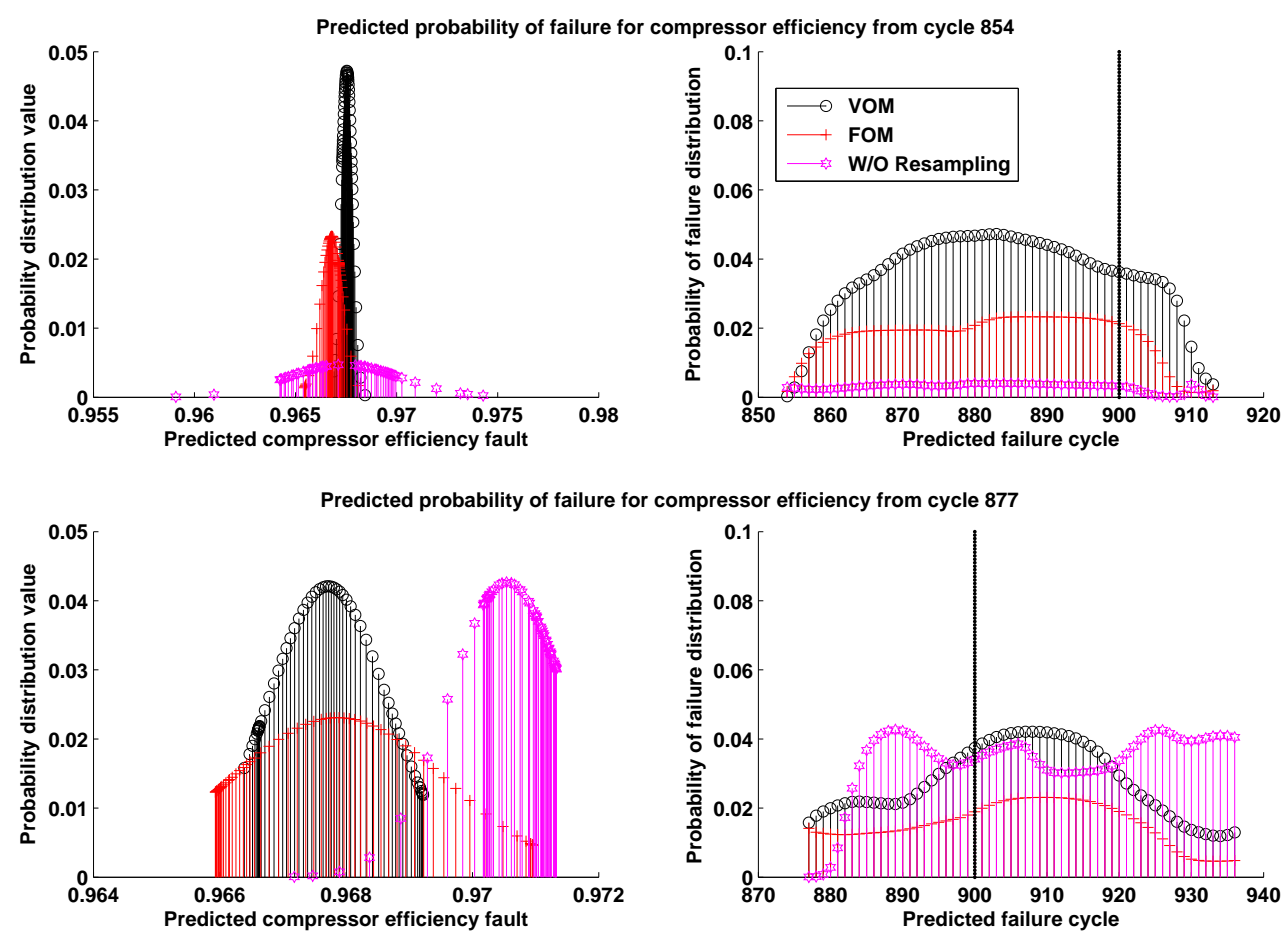

Fig. 6. Predicted probability of the failure for the compressor efficiency from two prediction windows.
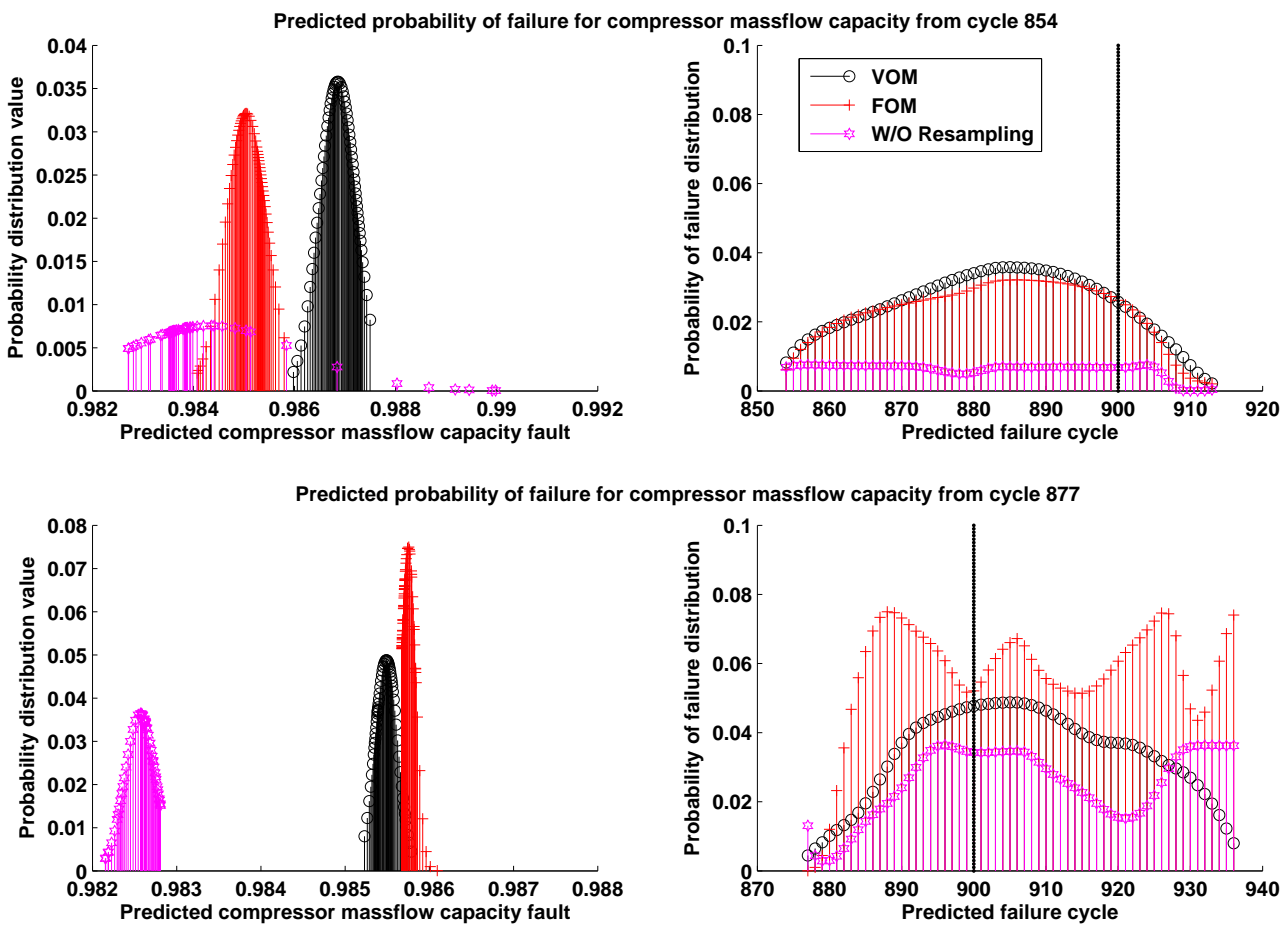

Fig. 7. Predicted probability of the failure for the compressor mass flow capacity from two prediction windows. 
TABLE XII

RUL PREDICTION IN COMPRESSOR FOULING SCENARIO

\begin{tabular}{|c|c|c|c|c||c|c|c||}
\hline \multirow{2}{*}{ Parameter } & \multicolumn{4}{|c||}{ Prediction from cycle 854 } & \multicolumn{3}{c||}{ Prediction from cycle 877 } \\
\cline { 2 - 8 } & method & failure cycle & predicted RUL & RUL error & failure cycle & predicted RUL & RUL error \\
\hline$\theta_{\eta_{C}}$ & VOM & 883 & 29 & +17 & 908 & 31 & -8 \\
& FOM & 888 & 34 & +12 & 910 & 33 & -10 \\
& W/O RS & 880 & 26 & +20 & - & - & - \\
\hline$\theta_{m_{C}}$ & VOM & 885 & 31 & +15 & 905 & 28 & -5 \\
& FOM & 886 & 32 & +14 & - & - & - \\
& W/O RS & - & - & - & 896 & 19 & +4 \\
\hline
\end{tabular}

for evaluating the failure cycle given the multiple system health parameters is based on the parameter that predicts an earlier failure cycle (according to the discussion in Section IV).

Erosion scenario: For the erosion scenario, the accomplished turbine health parameters prediction from two subsequent time windows close to the failure cycle are considered. These time windows start from the flight cycles 1114 and 1138, respectively. The health indicator vector in this scenario consists of the turbine efficiency and the mass flow capacity $\mathrm{HI}_{t+1: t+k}=\left(\begin{array}{ccc}\hat{\theta}_{\eta_{\mathrm{T} t+1 \mid t}} & \cdots & \hat{\theta}_{\eta_{\mathrm{T} t+k \mid t}} \\ \hat{\theta}_{m_{\mathrm{T} t+\ell \mid t}} & \cdots & \hat{\theta}_{m_{\mathrm{T} t+k \mid t}}\end{array}\right)$. The probability analysis similar to the fouling scenario is also performed for this scenario assuming the turbine health parameters as the system health indicators. However, considering that the erosion phenomenon is identified by its decreasing effect on the turbine efficiency and increasing effect on its mass flow capacity, the upper bound on the critical value of the turbine efficiency and the lower bound on the critical value of the turbine mass flow capacity are considered as the criteria for determining the system failure cycle. Consequently, in obtaining the failure cycle in this scenario the following criteria is considered,

$$
\begin{aligned}
& \text { FailureCycle }_{\eta_{\mathrm{T}}}=t+j \text {, such that } \hat{\theta}_{\eta_{\mathrm{T} t+j \mid t}} \leq(1.05) \theta_{\eta_{\mathrm{T}}}^{\text {cr }} \\
& \text { and } t+j=\operatorname{argmax}_{j=1}^{j=k} \operatorname{Gpdf}\left(\hat{\theta}_{\eta_{\mathrm{T}_{t+j \mid t}}}\right) \text {, } \\
& \text { FailureCycle }_{m_{\mathrm{T}}}=t+l \text {, such that } \hat{\theta}_{m_{\mathrm{T} t+l \mid t}} \geq(0.995) \theta_{m_{\mathrm{T}}}^{\mathrm{cr}} \\
& \text { and } t+l=\operatorname{argmax}_{l=1}^{l=k} \operatorname{Gpdf}\left(\hat{\theta}_{m_{\mathrm{T} t+l \mid t}}\right) \text {, }
\end{aligned}
$$

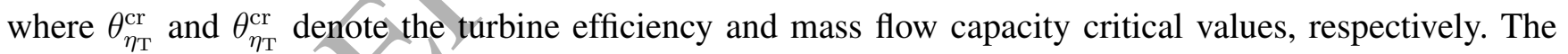
results that are summarized in Table XIII and Figures 8 and 9 are obtained based on the probabilistic analysis of the prediction results. The ground truth failure cycle is the cycle 1162 that corresponds to $\theta_{\eta_{\mathrm{T}}}^{\text {cr }}=0.94$ and $\theta_{m_{\mathrm{T}}}^{\text {cr }}=1.03$. The results presented in Table XIII show that from both time windows the distributions means related to the turbine fault parameters are located within the $99.5 \%$ confidence bound around the critical value for the turbine efficiency fault parameter. However, the FOM and the W/O RS do not result in prediction distributions within the $99.5 \%$ confidence bounds. According to the results indicated in Figures 8 and 9, the VOM method can be used for failure cycle prediction from both windows for both turbine health parameters, whereas the FOM cannot be used for this purpose from the first time window for the turbine efficiency and the W/O RS can not be used from the first time window for the turbine mass flow capacity.

Consequently, the predicted RUL from the starting point of the two considered time windows in the erosion scenario is calculated and presented in Table XIV. Since the actual failure cycle is located at 1162 , the actual RUL in the first time window starting at 1114 is $48(1162-1114=48)$, and in the second 

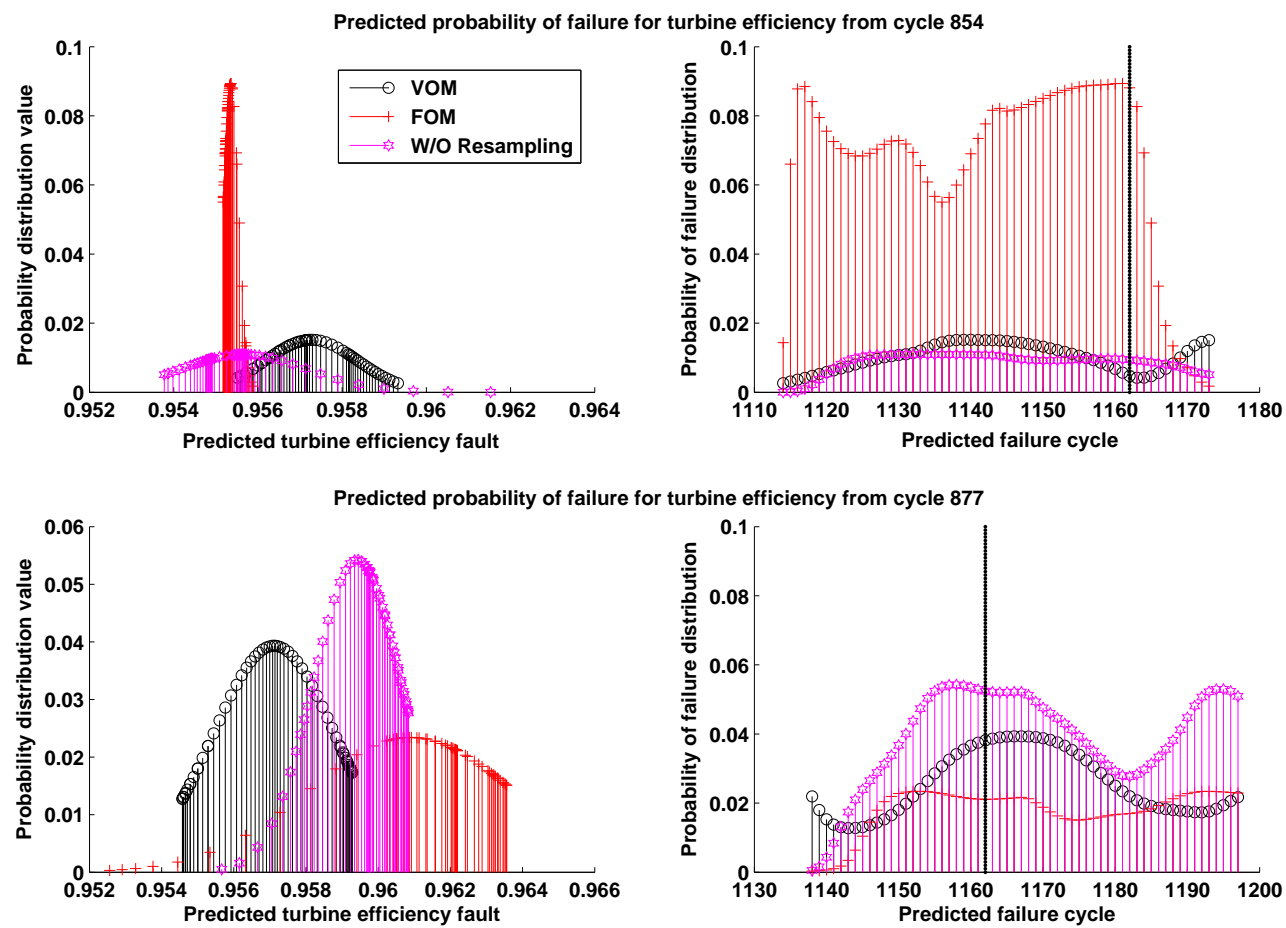

Fig. 8. Predicted probability of the failure for the turbine efficiency from two prediction windows.
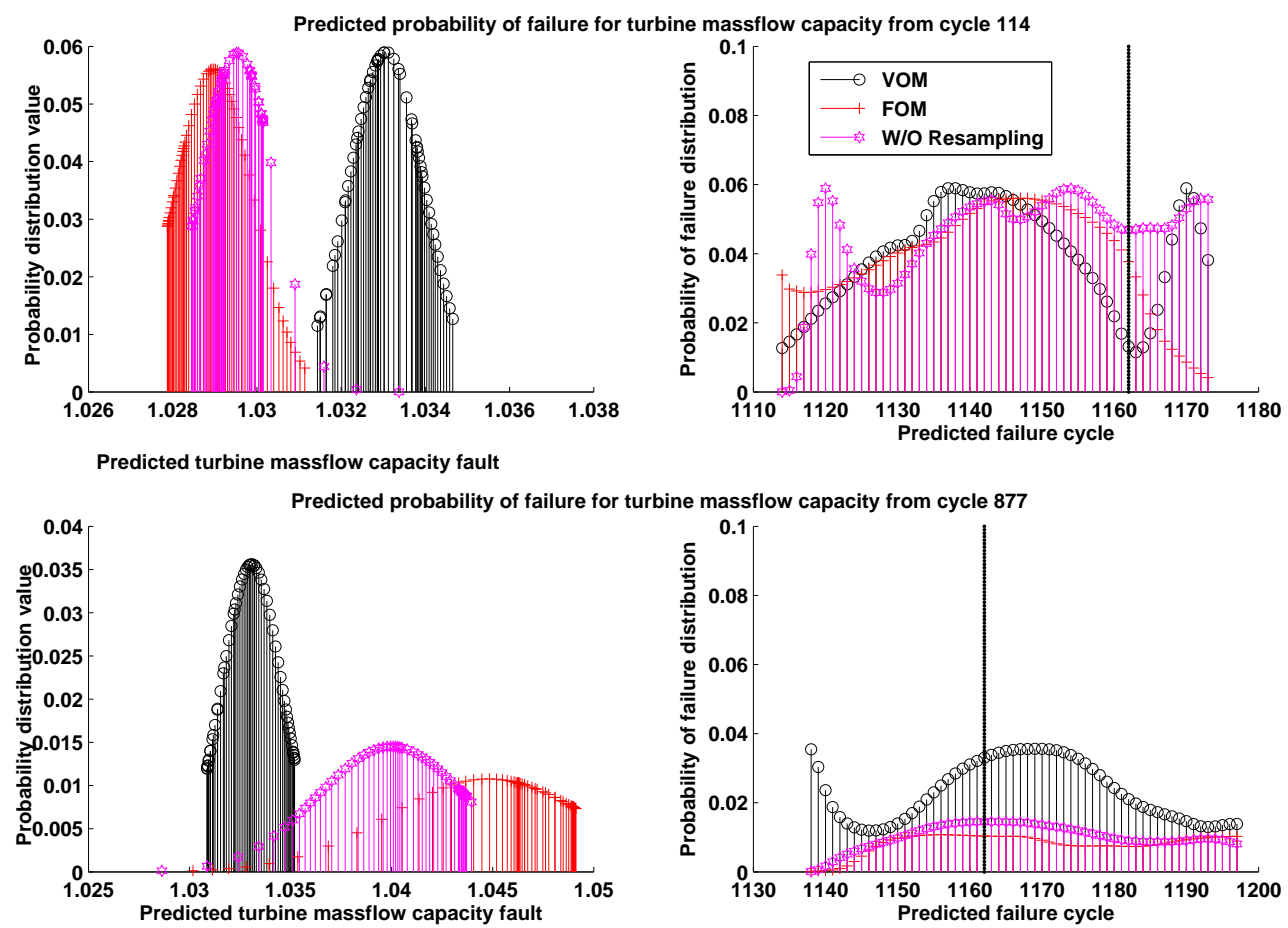

Fig. 9. Predicted probability of the failure for the turbine mass flow capacity from two prediction windows. 
TABLE XIII

PREDICTED DisTRIBUTIONS DUE TO THE TURBINE EROSION.

\begin{tabular}{|c|c|c|c||c|c||c|}
\hline \multirow{2}{*}{ Parameter } & \multicolumn{2}{|c||}{ Prediction from cycle 1114} & \multicolumn{2}{c||}{ Prediction from cycle 1138} & \multirow{2}{*}{ within $\pm 99.5 \%$ of critical value } \\
\cline { 2 - 6 } & method & mean & \pm std & mean & \pm std & yes \\
\hline$\theta_{\eta_{T}}$ & VOM & 0.957 & 0.0011 & 0.957 & 0.0017 & yes \\
& FOM & 0.955 & 0.0002 & 0.961 & 0.0028 & yes \\
\hline & W/O RS & 0.956 & 0.0015 & 0.959 & 0.0012 & yes \\
$\theta_{m_{T}}$ & VOM & 1.033 & $9.013 e^{-4}$ & 1.033 & 0.0015 & no \\
& FOM & 1.029 & $9.486 e^{-4}$ & 1.045 & 0.0049 & no \\
& W/O RS & 1.029 & $9.033 e^{-4}$ & 1.040 & 0.0037 & \\
\hline
\end{tabular}

TABLE XIV

RUL PREDICTION IN TURBINE EROSION SCENARIO

\begin{tabular}{|c|c|c|c|c||c|c|c||}
\hline \multirow{2}{*}{ Parameter } & \multicolumn{4}{|c||}{ Prediction from cycle 1114 } & \multicolumn{3}{c||}{ Prediction from cycle 1138 } \\
\cline { 2 - 8 } & method & failure cycle & predicted RUL & RUL error & failure cycle & predicted RUL & RUL error \\
\hline$\theta_{\eta_{T}}$ & VOM & 1139 & 25 & +23 & 1167 & 29 & -5 \\
& FOM & - & - & - & 1153 & 15 & +9 \\
& W/O RS & 1134 & 20 & +28 & 1158 & 20 & +4 \\
\hline$\theta_{m_{T}}$ & VOM & 1170 & 56 & -8 & 1170 & 32 & -8 \\
& FOM & 1147 & 33 & +15 & 1156 & 18 & +6 \\
& W/O RS & - & - & - & 1162 & 24 & 0 \\
\hline
\end{tabular}

time window starting at 1138 , the actual RUL is $24(1162-1138=24)$. The RUL error is also indicated in this table. From the results presented in Table XIV one can conclude that the RUL prediction using the FOM and the W/O RS according to variations in both health parameters of the turbine is possible only from the second window, i.e. as one gets closer to the failure cycle, whereas the VOM is capable of predicting the RUL based on both turbine health parameters from both time windows. However, as the time window moves towards the actual failure cycle, the RUL prediction based on the VOM becomes more accurate such that in the second time window the RUL can be predicted within \pm 5 cycles from the actual failure cycle. Moreover, the FOM and the W/O RS can determine the RUL based on both turbine health parameters only from the second window within \pm 9 and \pm 4 cycles from the actual failure cycle, respectively.

\section{RUL Performance Analysis with Different DLM Parameters}

In this subsection, performance of the RUL prediction scheme is evaluated when the parameters in the DLM are varying around their designed values for the prognosis of the gas turbine engine under both fouling and erosion scenarios. It should be pointed out that the designed parameters are selected as follows: the DLM fixed lag window size $\tau=150$, where 35 data points are used in the validation step $(s=35)$, the sliding window size in the M-distance algorithm is $\dot{q}=10$, the number of recent available observation at each time instant is selected as $s=10$, and the threshold in the M-distance for change detection is $\delta=1.5$. To evaluate the performance of prognosis in terms of the RUL prediction error, several scenarios are performed where in each scenario only one of the above mentioned parameters has been changed while the remainder of the DLM tuning parameters are considered to be fixed at their designed values. Moreover, the parameter $\iota=0.01$ is selected fixed for all the scenarios to obtain the maximum $k$-step ahead prediction horizon. This will satisfy the desired threshold on the $\operatorname{PRSME}_{y_{j}}$ value (based on 


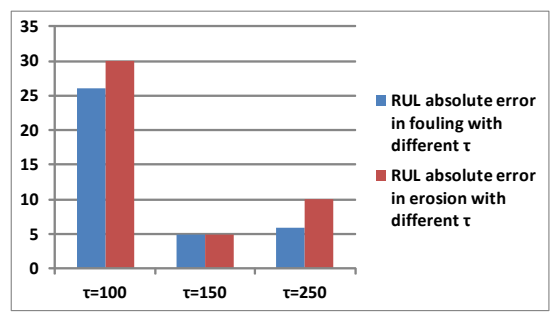

(a) RUL error subject to different $\tau$ values.

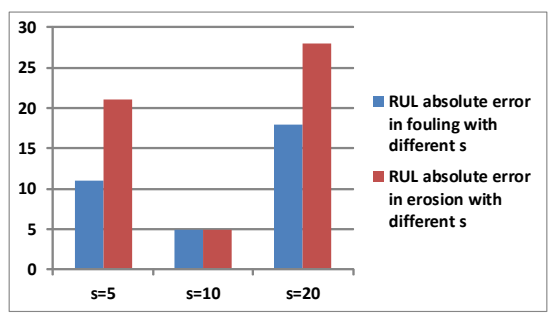

(c) RUL error subject to different $s$ values.

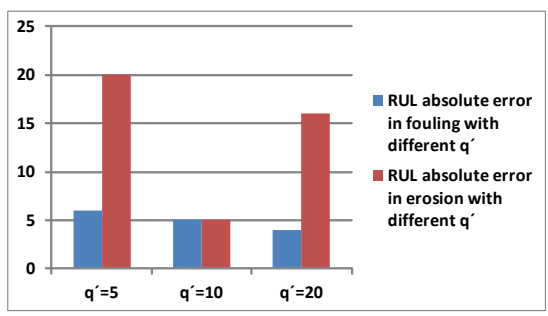

(b) RUL error subject to different $q$ ' values.

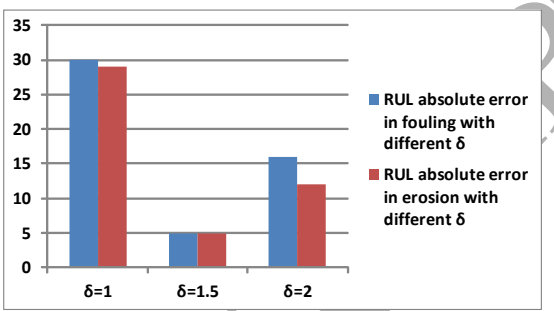

(d) RUL error subject to different $\delta$ values.

Fig. 10. RUL performance subject to selection of different DLM parameters.

८) in all considered scenarios that result in a maximum 30-steps ahead prediction horizon. Therefore, all comparisons among the various conditions are conducted in a prediction window of 30-steps before the failure occurrence.

In Figures 10 (a)-(d) the effects on changes of the DLM parameters in the RUL prediction absolute error are plotted for two more values around the selected parameter designed value. The summarized results are obtained from a window that is located in the 30-steps before reaching the ground truth failure cycle in each scenario. The presented results in Figure 10 (a) show that by decreasing the number of data points used in constructing the DLM model $(\tau)$ result in higher error in the RUL prediction in both fouling and erosion scenarios. From Figure 10 (b) one can conclude that whereas changes in the sliding window $q$ do not affect the RUL error of the fouling scenario significantly, the error due to the erosion scenario is rather high when $q$ is selected as a small or as a high value compared to the number of recent available data in each time instant, s. From the presented graphs in Figure 10 (c) one can also conclude that over increasing/decreasing the length of $s$ can affect the RUL error significantly in both scenarios. Finally, the results related to changes in the threshold which is utilized in the M-distance algorithm for change detection as presented in Figure 10 (d) show that selecting smaller values for $\delta$ can lead to erroneous RUL prediction results.

\section{E. Time Complexity Analysis of the Prediction Scheme}

To compare the efficiency of our proposed and developed prediction schemes based on the variable order DLM (VOM) and the fixed order DLM (FOM) models with another conventional method that is based on augmented state and parameter estimation algorithm with regularized particle filters and without resampling (W/O RS) [5], the execution times of theses schemes are now obtained for each iteration of the prediction step. Assuming that the computational complexity of the prediction step is proportional to the EF complexity of the algorithm [30]. These metrics are estimated by using an Intel Xeon CPU E31230, 
3.2 $\mathrm{GHz}$ processor with $16 \mathrm{~GB}$ memory. Therefore, the time complexity as a measure of time (in seconds) that are required to execute the algorithms for the best scenario (that is the minimum execution time), the average scenario (that is the average execution time), and the worst scenario (that is the maximum execution time) are obtained for the VOM, the FOM and the W/O RS schemes in Table XV. For all these methods the number of particles are considered to be the same and is set to $N_{0}=N=150$.

From the results that are shown, it can be concluded that the time required for execution of the VOM is less than that of the FOM and the W/O RS. It is further concluded that even with equal number of particles when the dimensions of the states and measurements are comparable with the dimension of the parameters ( $n_{\theta}=n_{x}=4, n_{y}=5$ ), the resampling algorithm that we have used (namely, the residual resampling) do not increase the time complexity (which is equivalent to the EF complexity) of our developed VOM algorithm when compared to a conventional method without performing resampling.

TABLE XV

Time COMPLEXITy ANALYSIS FOR THE VOM, FOM AND W/O RS IN SECONDS.

\begin{tabular}{|c|c|c|c|}
\hline Model & Best Scenario & Average Scenario & Worst Scenario \\
\hline VOM & 88 & 136.0208 & 190 \\
FOM & 94.0146 & 138.9729 & 194.5736 \\
$W / O R S$ & 92.4846 & 138.2435 & 193.9752 \\
\hline
\end{tabular}

\section{CONCLUSIONS}

In this paper, conventional particle filtering based schemes are extended to predict the future behavior of a nonlinear dynamical system states and parameters by utilizing the observation forecasting concept and time-series methods. The observation forecasting scheme is developed based on fixed/variable order DLM models that are being adjusted online according to an adaptive external adjustment loop. It is shown that despite its improved performance, our proposed scheme does not impose additional computational complexity when compared to other available methods in the literature. Our developed methodology is then applied for the purpose of failure prognosis of an aircraft gas turbine engine. The results of the remaining useful life (RUL) prediction do clearly demonstrate and illustrate the acceptable performance of our developed schemes.

\section{APPENDIX A}

PROOFS

\section{Proof of the Lemma 1:}

Let us define $S=\sum_{i=0}^{k-1} A^{i} B\left(A^{i}\right)^{\mathrm{T}}$. Given that $\left\|A^{i}\right\|=\left\|\left(A^{i}\right)^{\mathrm{T}}\right\|$, one gets

$$
\|S\| \leq\|B\| \sum_{i=1}^{k}\left\|A^{i}\right\|^{2} .
$$

Assuming that $\left\|A^{i}\right\|^{2} \leq \kappa r^{i}$, the summation in (23) represents a geometric series with common ratio that is less than one $(r<1)$. Therefore, it follows that $\sum_{i=1}^{k}\left\|A^{i}\right\|^{2} \leq \sum_{i=0}^{k-1} \kappa r^{i}=\frac{\kappa\left(1-r^{k}\right)}{1-r}$. Consequently, equation (23) can be re-written as

$$
\|S\| \leq\|B\| \frac{\kappa\left(1-r^{k}\right)}{1-r}
$$

where $\|B\| \frac{\kappa\left(1-r^{k}\right)}{1-r}$ is an upper bound of $\left\|\sum_{i=0}^{k-1} A^{i} B\left(A^{i}\right)^{\mathrm{T}}\right\|$ which is a function of $k$. This completes the proof of the lemma. 


\section{Proof of Theorem 1:}

Consider the state space representation of the ARMA model corresponding to each output that is represented as a univariate time-series having the DLM structure. The MSE of the $k$-step ahead forecast of the $j$-th output after utilizing $y_{j, t+k}^{\text {lin }}$ and $\hat{Y}_{j, t+k \mid t}$ from (12) and (13), respectively, can be obtained as follows. First, we have

$$
\mathbb{E}\left\{\left(y_{j, t+k}^{\operatorname{lin}}-\hat{Y}_{j, t+k \mid t}\right)^{2}\right\}=\mathbb{E}\left\{\left(H\left(\sum_{i=0}^{\infty}\left(G_{j, l}\right)^{i} F_{j, \tau} \varepsilon_{j, t+k-i}-\left(G_{j, l}\right)^{k} \sum_{i=0}^{\infty}\left(G_{j, l}\right)^{i} F_{j, l} \varepsilon_{j, t-i}\right)\right)^{2}\right\} .
$$

Expanding the term inside the expectation in the right hand side of (25) results in $y_{j, t+k}^{\operatorname{lin}}-\hat{Y}_{j, t+k \mid t}=$ $H\left(F_{j, l} \varepsilon_{j, t+k}+G_{j, l} F_{j, l} \varepsilon_{j, t+k-1}+\ldots+\left(G_{j, l}\right)^{k-1} F_{j, l} \varepsilon_{j, t+1}+\left(G_{j, l}\right)^{k} F_{j, l} \varepsilon_{j, t}+\ldots-\left(G_{j, l}\right)^{k} F_{j, l} \varepsilon_{j, t}-\left(G_{j, l}\right)^{k+1} F_{j, l} \varepsilon_{j, t-1}-\right.$ $\ldots)=H\left(\sum_{i=0}^{k-1}\left(G_{j, l}\right)^{i} F_{j, l} \varepsilon_{j, t+k-i}\right)$. Hence, the mean-square error can be written as $\mathbb{E}\left\{\left(y_{j, t+k}-\hat{Y}_{j, t+k \mid t}\right)^{2}\right\}=$ $\mathbb{E}\left\{H\left(F_{j, l} \varepsilon_{j, t+k}+G_{j, l} F_{j, l} \varepsilon_{j, t+k-1}+\ldots+\left(G_{j, l}\right)^{k-1} F_{j, l} \varepsilon_{j, t+1}\right) \times\left(F_{j, l} \varepsilon_{j, t+k}+G_{j, l} F_{j, l} \varepsilon_{j, t+k-1}+\ldots\right.\right.$

$\left.\left.+\left(G_{j, l}\right)^{k-1} F_{j, l} \varepsilon_{j, t+1}\right)^{\mathrm{T}} H^{\mathrm{T}}\right\}$. Consequently, since $\mathbb{E}\left\{\varepsilon_{j, t-n} \varepsilon_{j, t-m}\right\}=\left\{\begin{aligned} \sigma_{\varepsilon_{j}}^{2}, & \text { if } n=m \\ 0, & \text { if } n \neq m\end{aligned}\right.$, we have

$$
\mathbb{E}\left\{\left(y_{j, t+k}^{\operatorname{lin}}-\hat{Y}_{j, t+k \mid t}\right)^{2}\right\}=\sigma_{\varepsilon_{j}}^{2} H\left(\sum_{i=0}^{k-1}\left(G_{j, l}\right)^{i} F_{j, l} F_{j, l}^{\mathrm{T}}\left(G_{j, l}\right)^{i^{\mathrm{T}}}\right) H^{\mathrm{T}} .
$$

By applying the result of Lemma 1 to the right hand side of (26), the following upper bound on the forecast error is obtained,

$$
\mathbb{E}\left\{\left(y_{j, t+k}^{\operatorname{lin}}-\hat{Y}_{j, t+k \mid t}\right)^{2}\right\} \leq \sigma_{\varepsilon_{j}}^{2}\left\|F_{j, l} F_{j, l}^{\mathrm{T}}\right\|\left(\frac{\kappa_{j}\left(1-r_{j}^{k}\right)}{1-r_{j}}\right) .
$$

Consequently, the upper bound of the MSE as $\mathbb{E}\left\{\left(y_{j, t+k}^{\operatorname{lin}}-\hat{Y}_{j, t+k \mid t}\right)^{2}\right\}$ is dependent on the prediction horizon $k$. This completes the proof of Theorem 1 .

\section{Proof of Theorem 2:}

Let us construct a vector of $k$-step ahead predicted observations that are obtained through $n_{y}$ univariate time-series and fixed-lag DLM models as $\hat{Y}_{t+k \mid t}=\left(\hat{Y}_{1, t+k \mid t}, \hat{Y}_{2, t+k \mid t}, \ldots, \hat{Y}_{n_{y}, t+k \mid t}\right)^{\mathrm{T}}$. At the time instant $t$ the forecasted observations (from the linear regression model of the ARMA process), $\hat{Y}_{j, t+k \mid t}$ are independent from one another since each observation is constructed only from its own historical data, i.e., $\operatorname{cov}\left(\hat{Y}_{n, t+k \mid t} \hat{Y}_{m, t+k \mid t}\right)=0$, for $n \neq m$. Consequently, the covariance matrix of $\hat{Y}_{t+k \mid t}$ reduces to the variance matrix with diagonal entities as $\operatorname{var}\left(\hat{Y}_{j, t+k \mid t}\right), j=1, \ldots, n_{y}$. The $k$-step ahead prediction error of the $j$-th observation, when it is obtained based on the fixed-lag DLM model using the ARMA process is approximated along the lines that are described in Theorem 1. In this theorem the nonlinear observation $y_{j, t}$ was approximated with its linear projection according to the ARMA process that was introduced in (3) and defined in Remark 4, i.e. at the time $t, y_{j, t} \approx y_{j, t}^{\operatorname{lin}}$. Therefore, the error due to mismatch between the actual nonlinear observation and its corresponding linear projection (12) in the $k$-step ahead prediction horizon is calculated as $\mathbb{E}\left\{\left(y_{j, t+k}-y_{j, t+k}^{\text {lin }}\right)^{2}\right\}=\mathbb{E}\left\{\left(y_{j, t+k}-\mu_{j, l}-H \sum_{i=0}^{\infty}\left(G_{j, l}\right)^{i} F_{j, l} \varepsilon_{j, t+k-i}\right)\left(y_{j, t+k}-\mu_{j, l}-\right.\right.$ $\left.\left.H \sum_{i=0}^{\infty}\left(G_{j, l}\right)^{i} F_{j, l} \varepsilon_{j, t+k-i}\right)^{\mathrm{T}}\right\}=\mathbb{E}\left\{y_{j, t+k}^{2}\right\}-2 \mathbb{E}\left\{y_{j, t+k} \mu_{j, l}\right\}-2 \mathbb{E}\left\{y_{j, t+k}\left(\sum_{i=0}^{\infty}\left(G_{j, l}\right)^{i} F_{j, l} \varepsilon_{j, t+k-i}\right)^{\mathrm{T}} H^{\mathrm{T}}\right\}+$ $\mathbb{E}\left\{\mu_{j, l}^{2}\right\}+2 \mathbb{E}\left\{\mu_{j, l}\left(\sum_{i=0}^{\infty}\left(G_{j, l}\right)^{i} F_{j, l} \varepsilon_{j, t+k-i}\right)^{\mathrm{T}} H^{\mathrm{T}}\right\}$

$+\mathbb{E}\left\{H\left(\sum_{i=0}^{\infty}\left(G_{j, l}\right)^{i} F_{j, l} \varepsilon_{j, t+k-i}\right)\left(\sum_{i=0}^{\infty}\left(G_{j, l}\right)^{i} F_{j, l} \varepsilon_{j, t+k-i}\right)^{\mathrm{T}} H^{\mathrm{T}}\right\}$.

Let us assume that the white noise process $\varepsilon_{j, t}$ and the measurement noise process $\nu_{t}$ in (2) are zero-mean process with covariance matrices $\sigma_{\varepsilon_{j, t}}^{2}$, and $V_{t}=\operatorname{diag}\left(\sigma_{\nu_{1, t}}^{2}, \cdots, \sigma_{\nu_{n_{y}, t}}^{2}\right)$, respectively. Moreover, $v_{t}$ and $\varepsilon_{j, t}$ 
are two independent processes which yields $\mathbb{E}\left\{y_{j, t} \epsilon_{j, t}\right\}=0, \forall t$, and $\mathbb{E}\left\{y_{j, t}\right\}=\mathbb{E}\left\{h_{t}\left(x_{t}, \theta_{t}^{\mathrm{T}} \lambda\left(x_{t}\right)\right)+\nu_{j, t}\right\}=$ $h_{t}\left(x_{t}, \theta_{t}^{\mathrm{T}} \lambda\left(x_{t}\right)\right)$. Furthermore, in view of the fact that $y_{j, t+k}$ is conditionally independent from $\epsilon_{j, t+k}$ (since $\epsilon_{j, t+k}$ is used to construct $y_{j, t+k}^{\operatorname{lin}}$ and not $\left.y_{j, t+k}\right)$, the above equation reduces to $\mathbb{E}\left\{\left(y_{j, t+k}-y_{j, t+k}^{\operatorname{lin}}\right)^{2}\right\}=$ $\sigma_{\nu_{j, t+k}}^{2}+h_{j, t+k}^{2}\left(x_{t+k}, \theta_{t+k}^{\mathrm{T}} \lambda\left(x_{t+k}\right)\right)-2 \mu_{j, l} h_{j, t+k}\left(x_{t+k}, \theta_{t+k}^{\mathrm{T}} \lambda\left(x_{t+k}\right)\right)-0+\sigma_{\varepsilon_{j}}^{2} H\left(\sum_{i=0}^{\infty}\left(G_{j, l}\right)^{i} F_{j, l} F_{j, l}^{\mathrm{T}}\left(G_{j, l}\right)^{i^{\mathrm{T}}}\right) H^{\mathrm{T}}$. Now, one requires to calculate the error due to the $k$-step ahead forecast of the observation vector according to the ARMA process, that is denoted by $\hat{Y}_{j, t+k \mid t}$ with the original nonlinear observations $y_{j, t+k}$, where $\hat{Y}_{j, t+k \mid t}$ is considered to be obtained from (13) in the selected time window as long as the $l$-th DLM is valid. Hence, by considering the linear projection $y_{j, t+k}^{\operatorname{lin}}$ for the $k$-step ahead prediction horizon, the expectation of the error between $y_{j, t+k}$ and $\hat{Y}_{j, t+k \mid t}$ can be expressed as,

$$
\mathbb{E}\left\{\left|y_{j, t+k}-\hat{Y}_{j, t+k \mid t}\right|\right\}=\mathbb{E}\left\{\left|y_{j, t+k}-y_{j, t+k}^{\operatorname{lin}}+y_{j, t+k}^{\operatorname{lin}}-\hat{Y}_{j, t+k \mid t}\right|\right\}
$$

Let us consider the MSE representation $E\left\{\left|y_{j, t+k}-y_{j, t+k}^{\operatorname{lin}}+y_{j, t+k}^{\operatorname{lin}}-\hat{Y}_{j, t+k \mid t}\right|^{2}\right\}$. The Minkowski's inequality [51] is now utilized to obtain the following inequality $\mathbb{E}\left\{\left|y_{j, t+k}-y_{j, t+k}^{\operatorname{lin}}+y_{j, t+k}^{\lim }-\hat{Y}_{j, t+k \mid t}\right|^{2}\right\} \leq \mathbb{E}\left\{\left(y_{j, t+k}-\right.\right.$ $\left.\left.\left.y_{j, t+k}^{\operatorname{lin}}\right)^{2}\right\}+\mathbb{E}\left\{\left(y_{j, t+k}^{\operatorname{lin}}-\hat{Y}_{j, t+k \mid t}\right)^{2}\right\}+2 \sqrt{\mathbb{E}\left\{y_{j, t+k}-y_{j, t+k}^{\operatorname{lin}}\right\}^{2} \mathbb{E}\left\{y_{j, t+k}^{\operatorname{lin}}-\hat{Y}_{j, t+k \mid t}\right\}^{2}}\right)$. Finally, the upper bound on the error is obtained as $E\left\{\left|y_{j, t+k}-\hat{Y}_{j, t+k \mid t}\right|^{2}\right\} \leq \mathbb{E}\left\{\left(y_{j, t+k} \tau y_{j, t+k}^{\operatorname{lin}}\right)^{2}\right\}+\mathbb{E}\left\{\left(y_{j, t+k}^{\operatorname{lin}}-\hat{Y}_{j, t+k \mid t}\right)^{2}\right\}+$ $2 \sqrt{\mathbb{E}\left\{y_{j, t+k}-y_{j, t+k}^{\operatorname{lin}}\right\}^{2} \mathbb{E}\left\{y_{j, t+k}^{\operatorname{lin}}-\hat{Y}_{j, t+k \mid t}\right\}^{2}} \leq \sigma_{y_{j, t}}^{2}+\mathcal{C}_{j, l}+\mathcal{D}_{j, l}+\mathcal{B}_{j, l}+2 \sqrt{\left(\sigma_{y_{j, t}}^{2}+\mathcal{C}_{j, l}+\mathcal{D}_{j, l}\right) \mathcal{B}_{j, l}}$, where $\mathcal{D}_{j, l}$ is obtained according to Lemma 1 as an upper bound on the last term in the right hand side of the first equation above. Moreover, $\mathcal{B}_{j, l}$ is an upper bound on the observation forecast error from the DLM model according to Theorem 1. Consequently, since for the dynamical system we have $\forall\left(x_{t}, \theta_{t}\right) \in \mathcal{D}_{\mathcal{R}}$, the function $h_{t}\left(x_{t}, \theta_{t}^{\mathrm{T}} \lambda\left(x_{t}\right)\right)$ and as a result $\mathcal{C}_{j, l}$ will be bounded. Consequently, the $k$-step ahead prediction error due to the observations will remain bounded. This completes the proof of the theorem.

\section{ACKNOWLEDGMENT}

The authors would like to express their thanks to the reviewers for their comments and suggestions that have improved the paper's readability and clarity.

\section{REFERENCES}

[1] R. Sekhon, H. Bassily, and J. Wagner, "A comparison of two trending strategies for gas turbine performance prediction," Journal of Engineering for Gas Turbines and Power, vol. 130, no. 4, p. 041601, 2008.

[2] A. Grall, L. Dieulle, C. Berenguer, and M. Roussignol, "Continuous-time predictive-maintenance scheduling for a deteriorating system," IEEE Transactions on Reliability, vol. 51, pp. 141-150, Jun 2002.

[3] H.-Q. Gu, S. Zhang, and L. Ma, "Process analysis for performance evaluation of prognostics methods orienting to engineering application," in International Conference on Quality, Reliability, Risk, Maintenance, and Safety Engineering, 2012.

[4] P. Shetty, D. Mylaraswamy, and T. Ekambaram, "A hybrid prognostic model formulation and health estimation of auxiliary power units," Journal of Engineering for Gas Turbines and Power, vol. 130, no. 2, pp. 021601-1-021601-9, 2008.

[5] M. E. Orchard and G. J. Vachtsevanos, "A particle-filtering approach for on-line fault diagnosis and failure prognosis," Transactions of the Institute of Measurement and Control, 2009.

[6] J. Son, Q. Zhou, S. Zhou, X. Mao, and M. Salman, "Evaluation and comparison of mixed effects model based prognosis for hard failure," IEEE Transactions on Reliability, vol. 62, no. 2, pp. 379-394, 2013.

[7] X.-S. Si, W. Wang, C.-H. Hu, D.-H. Zhou, and M. G. Pecht, "Remaining useful life estimation based on a nonlinear diffusion degradation process," IEEE Transactions on Reliability, vol. 61, no. 1, pp. 50-67, 2012.

[8] D. Simon and D. L. Simon, "Aircraft turbofan engine health estimation using constrained Kalman filtering," Journal of engineering for Gas Turbines and Power, vol. 127, no. 2, pp. 323-328, 2005.

[9] P. Baraldi, F. Mangili, and E. Zio, "A Kalman filter-based ensemble approach with application to turbine creep prognostics," IEEE Transactions on Reliability, vol. 61, no. 4, pp. 966-977, 2012. 
[10] B. Pattipati, C. Sankavaram, K. Pattipati, Y. Zhang, M. Howell, and M. Salman, "Multiple model moving horizon estimation approach to prognostics in coupled systems," in AUTOTESTCON, 2011.

[11] J. Sun, H. Zuo, and M. Pecht, "Advances in sequential Monte Carlo methods for joint state and parameter estimation applied to prognostics," in Prognostics and System Health Management Conference (PHM-Shenzhen), 2011.

[12] B. E. Olivares, C. Muñoz, M. E. Orchard, and J. F. Silva, "Particle-filtering-based prognosis framework for energy storage devices with a statistical characterization of state-of-health regeneration phenomena," IEEE Transactions on Instrumentation and Measurement, vol. 62, no. 2, pp. 364-376, 2013.

[13] N. Daroogheh, N. Meskin, and K. Khorasani, "A novel particle filter parameter prediction scheme for failure prognosis," in Proceedings of the American Control Conference, pp. 1735-1742, 2014.

[14] N. Daroogheh, N. Meskin, and K. Khorasani, "Particle filtering for state and parameter estimation in gas turbine engine fault diagnostics," in Proceedings of the American Control Conference, pp. 4343-4349, 2013.

[15] N. Daroogheh, N. Meskin, and K. Khorasani, "A dual particle filter-based fault diagnosis scheme for nonlinear systems," in IEEE Transactions on Control Systems Technology, p. DOI: 10.1109/TCST.2017.2705056, 2017.

[16] N. Daroogheh, N. Meskin, and K. Khorasani, "Particle filters for dual state and parameter estimation of nonlinear systems with application to fault diagnosis of gas turbine engines," https://archive.org/details/DualParticleFilter, 2014.

[17] M. West, "Mixture models, Monte Carlo, Bayesian updating, and dynamic models," Computing Science and Statistics, pp. 325-325, 1993.

[18] J. D. Hamilton, Time series analysis, vol. 2. Princeton university press Princeton, 1994.

[19] D. A. Pola, H. F. Navarrete, M. E. Orchard, R. S. Rabie, M. A. Cerda, B. E. Oliyares, J. F. Silva, P. A. Espinoza, and A. Perez, "Particle-filtering-based discharge time prognosis for lithium-ion batteries with a statistical characterization of use profiles," IEEE Transactions on Reliability, vol. 64, no. 2, pp. 710-720, 2015.

[20] M. West and J. Harrison, "Bayesian forecasting and dynamic models," vol. 18, 1997.

[21] A. Soylemezoglu, S. Jagannathan, and C. Saygin, "Mahalanobis-taguchi system as a multi-sensor based decision making prognostics tool for centrifugal pump failures," IEEE Transactions on Reliability, vol. 60, no. 4, pp. 864-878, 2011.

[22] M. Orchard, G. Kacprzynski, K. Goebel, B. Saha, and G. Vachtsevanos, "Advances in uncertainty representation and management for particle filtering applied to prognostics," in International Conference on Prognostics and Health Management, 2008.

[23] M. Orchard, F. Tobar, and G. Vachtsevanos, "Outer feedback correction loops in particle filtering-based prognostic algorithms: Statistical performance comparison," Studies in Informatics and Control, vol. 18, no. 4, pp. 295-304, 2009.

[24] M. Orchard, L. Tang, B. Saha, K. Goebel, and G. Nachtsevanos, "Risk-sensitive particle-filtering-based prognosis framework for estimation of remaining useful life in energy storage devices," Studies in Informatics and Control, vol. 19, no. 3, pp. 209-218, 2010.

[25] A. Saxena, J. Celaya, B. Saha, S. Saha, and K. Goebel, "Metrics for offline evaluation of prognostic performance," International Journal of Prognostics and Health Management, vol. 1, p. 4, 2010.

[26] A. Saxena, J. Celaya, E. Balaban, K. Goebel, B. Saha, S. Saha, and M. Schwabacher, "Metrics for evaluating performance of prognostic techniques," in International Conference on Prognostics and Health Management, 2008.

[27] S. Uckun, K. Goebel, and P. Lucas, "Standardizing research methods for prognostics," in International Conference on Prognostics and Health Management, 2008.

[28] X. Guan, Y. Liu, R. Jha, A. Saxena, J. Celaya, and K. Geobel, "Comparison of two probabilistic fatigue damage assessment approaches using prognostic performance metrics," International Journal of the PHM Society, vol. 1, no. 005, 2011.

[29] M. Daigle and K. Goebel, "Multiple damage progression paths in model-based prognostics," in Aerospace Conference, IEEE, 2011.

[30] R. Karlsson, T. Schön, and F. Gustafsson, "Complexity analysis of the marginalized particle filter," IEEE Transactions on Singnal Processing, vol. 53, no. 11, pp. 4408-4411, 2005.

[31] E. Naderi, N. Meskin, and K. Khorasani, "Nonlinear fault diagnosis of jet engines by using a multiple model-based approach," Journal of Engineering for Gas Turbines and Power, vol. 134, no. 1, p. 011602, 2012.

[32] N. Meskin, E. Naderi, and K. Khorasani, "A multiple model-based approach for fault diagnosis of jet engines," IEEE Transactions on Control Systems Technology, vol. 21, no. 1, 2013.

[33] R. Mohammadi, E. Naderi, K. Khorasani, and S. Hashtrudi-Zad, "Fault diagnosis of gas turbine engines by using dynamic neural networks," ASME Conference Proceedings, vol. 2010, no. 43987, pp. 365-376, 2010.

[34] W. P. J. Visser, O. Kogenhop, and M. Oostveen, "A generic approach for gas turbine adaptive modeling," Journal of Engineering for Gas Turbines and Power, vol. 128, no. 1, pp. 13-19, 2006.

[35] M. Naeem, "Impacts of low-pressure (lp) compressors fouling of a turbofan upon operational- effectiveness of a military aircraft," Applied Energy, vol. 85, no. 4, pp. 243-270, 2008.

[36] N. Daroogheh, A. Vatani, M. Gholamhossein, and K. Khorasani, "Engine life evaluation based on a probabilistic approach," in ASME 2012 International Mechanical Engineering Congress and Exposition, pp. 347-358, 2012.

[37] A. C. Harvey, Forecasting, Structural Time Series Models and the Kalman Filter. Cambridge University Press, 1990.

[38] G. Verdier and A. Ferreira, "Adaptive mahalanobis distance and k -nearest neighbor rule for fault detection in semiconductor manufacturing," IEEE Transactions on Semiconductor Manufacturing, vol. 24, no. 1, pp. 59-68, 2011. 
[39] L. Ljung, System Identification. Wiley Online Library, 1999.

[40] S. Jaggia, "Forecasting with ARMA models," Case Studies In Business, Industry And Government Statistics, vol. 4, no. 1, pp. 59-65, 2014.

[41] L. Ljung, "System Identification: Theory for the User," Prentice Hall Information and System Sciences Series, New Jersey, vol. 7632, 1987.

[42] E. Ahmed, A. Clark, and G. Mohay, "A novel sliding window based change detection algorithm for asymmetric traffic," in International Conference on Network and Parallel Computing,, pp. 168-175, IEEE, 2008.

[43] J. D. Hamilton, Time Series Analysis, vol. 2. Princeton university press Princeton, 1994.

[44] R. Smith, "Matrix equation xa+bx=c," SIAM Journal on Applied Mathematics, vol. 16, no. 1, pp. 198-201, 1968.

[45] N. Oudjane and C. Musso, "Progressive correction for regularized particle filters," in Proceedings of the Third International Conference on Information Fusion, vol. 2, pp. THB2-10, IEEE, 2000.

[46] N. Daroogheh, A. Baniamerian, N. Meskin, and K. Khorasani, "Prognosis and health monitoring of nonlinear systems using a hybrid scheme through integration of pfs and neural networks," IEEE Transactions on Systems, Man, and Cybernetics: Systems, vol. 47, no. 8, pp. 1990-2004, 2017.

[47] M. Daigle, I. Roychoudhury, S. Narasimhan, S. Saha, B. Saha, and K. Goebel, "Investigating the effect of damage progression model choice on prognostics performance," in Proceedings of the Annual Conference of the Prognostics and Health Management Society, 2011.

[48] A. Doucet, S. Godsill, and C. Andrieu, "On sequential Monte Carlo sampling methods for Bayesian filtering," Statistics and computing, vol. 10, no. 3, pp. 197-208, 2000.

[49] M. Naeem, R. Singh, and D. Probert, "Implications of engine's deterioration upon an aero-engine hp turbine blade's thermal fatigue life," International journal of fatigue, vol. 22, 2000.

[50] Y. Li and P. Nilkitsaranont, "Gas turbine performance prognostic for condition-based maintenance," Applied Energy, vol. 86, pp. 2152$2161,2009$.

[51] G. Hardy, J. Littlewood, and G. Polya, "Inequalities. reprint of the 1952 edition. cambridge mathematical library,” 1988. 